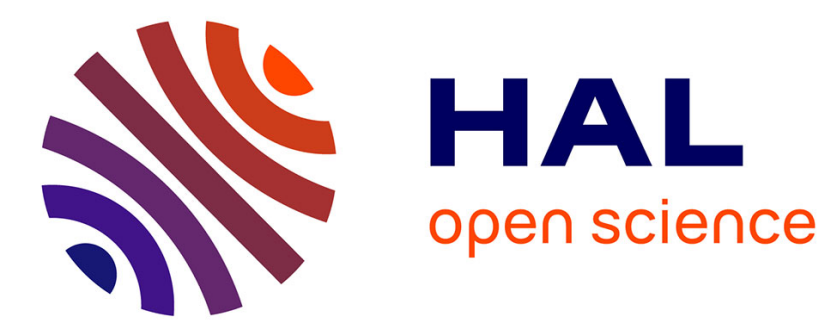

\title{
Further Results on an Equitable 1-2-3 Conjecture
}

Julien Bensmail, Foivos Fioravantes, Fionn Mc Inerney, Nicolas Nisse

\section{To cite this version:}

Julien Bensmail, Foivos Fioravantes, Fionn Mc Inerney, Nicolas Nisse. Further Results on an Equitable 1-2-3 Conjecture. Discrete Applied Mathematics, 2021, 297, pp.1-20. 10.1016/j.dam.2021.02.037 Get . hal-02533537v2

\section{HAL Id: hal-02533537 \\ https://hal.science/hal-02533537v2}

Submitted on 2 Feb 2021 (v2), last revised 21 Feb 2022 (v3)

HAL is a multi-disciplinary open access archive for the deposit and dissemination of scientific research documents, whether they are published or not. The documents may come from teaching and research institutions in France or abroad, or from public or private research centers.
L'archive ouverte pluridisciplinaire HAL, est destinée au dépôt et à la diffusion de documents scientifiques de niveau recherche, publiés ou non, émanant des établissements d'enseignement et de recherche français ou étrangers, des laboratoires publics ou privés. 


\title{
Further Results on an Equitable 1-2-3 Conjecture*
}

\author{
Julien Bensmail ${ }^{1}$, Foivos Fioravantes ${ }^{1}$, Fionn Mc Inerney ${ }^{2}$, and Nicolas Nisse ${ }^{1}$ \\ ${ }^{1}$ Université Côte d'Azur, Inria, CNRS, I3S, France \\ ${ }^{2}$ Laboratoire d'Informatique et Systèmes, Aix-Marseille Université, CNRS, and Université de Toulon \\ Faculté des Sciences de Luminy, Marseille, France
}

\begin{abstract}
In this work, we consider equitable proper labellings of graphs, which were recently introduced by Baudon, Pilśniak, Przybyło, Senhaji, Sopena, and Woźniak. Given a graph G, the goal is to assign labels to the edges so that 1) no two adjacent vertices are incident to the same sum of labels, and 2) every two labels are assigned about the same number of times. Particularly, we aim at designing such equitable proper $k$-labellings of $G$ with $k$ being as small as possible. In connection with the so-called 1-2-3 Conjecture, it might be that labels 1,2,3 are, a few obvious exceptions apart, always sufficient to achieve this just as in the non-equitable version of the problem.

We provide results regarding some open questions about equitable proper labellings. Via a hardness result, we first prove that there exist infinitely many graphs for which more labels are required in the equitable version than in the non-equitable version. This remains true in the bipartite case. We finally show that, for every $k \geq 3$, every $k$-regular bipartite graph admits an equitable proper $k$-labelling.
\end{abstract}

Keywords: Proper labellings, Equitable labellings, 1-2-3 Conjecture.

\section{Introduction}

This paper deals with a variant of the so-called 1-2-3 Conjecture, which is defined through the following notions. Consider a (simple undirected) graph $G$. Let us consider a $k$-labelling $\ell$ of $G$ to be an assignment $\ell: E(G) \rightarrow\{1, \ldots, k\}$ of labels to the edges of $G$. For each vertex $v$ of $G$, one can compute, as a colour $c_{\ell}(v)$ of $v$, the sum of the labels assigned by $\ell$ to the edges incident to $v$. We say that $\ell$ is proper if $c_{\ell}$ is indeed a proper vertex-colouring of $G$, i.e., we have $c_{\ell}(u) \neq c_{\ell}(v)$ for every edge $u v \in E(G)$. In this field, we say that $G$ is nice if it has no connected component isomorphic to $K_{2}$. This is because $K_{2}$ is indeed the only connected graph with no proper labelling. In other words, the parameter $\chi_{\Sigma}(G)$, which is the minimum $k$ such that $G$ admits a proper $k$-labelling, is defined for every nice graph $G$. Actually, Karoński, Łuczak, and Thomason conjectured in 2004 that $\chi_{\Sigma}(G)$ should never exceed 3 (see [6]):

Conjecture 1.1 (1-2-3 Conjecture). If $G$ is a nice graph, then $\chi_{\Sigma}(G) \leq 3$.

A few facts on the 1-2-3 Conjecture are worth mentioning. In particular, the upper bound in the statement of the conjecture cannot be decreased to 2 in general, as shown for instance by complete

\footnotetext{
${ }^{*}$ This work has been partially supported by the ANR project DISTANCIA (ANR-14-CE25-0006).
} 
graphs and odd-length cycles. More generally, it is known that, given a nice graph $G$, deciding whether $\chi_{\Sigma}(G) \leq 2$ holds is NP-complete (thus, unless $\mathrm{P}=\mathrm{NP}$, there is no "good" characterisation of graphs admitting proper 2-labellings) [4]. This contrasts with the bipartite case, however, as it was more recently shown that bipartite graphs $G$ with $\chi_{\Sigma}(G)=3$ form a restricted family of graphs [12]. To date, the best result towards the 1-2-3 Conjecture was established by Kalkowski, Karoński, and Pfender in [5], who proved that $\chi_{\Sigma}(G) \leq 5$ holds for every nice graph $G$. We refer the interested reader to [10] for a survey by Seamone on this topic.

Several side aspects of the 1-2-3 Conjecture are of interest, such as the importance of the three labels 1,2,3 for designing proper labellings. In particular, a total version of the 1-2-3 Conjecture introduced by Przybyło and Woźniak in [9], where also the vertices must be labelled, seems to indicate that, in general, for any nice graph we should be able to design 2-labellings that are close to proper. As a consequence, to the best of our knowledge there is no known (non-trivial) graph $G$ with $\chi_{\Sigma}(G)=3$ for which we must use the three labels $1,2,3$ with nearly equal proportion in every proper 3-labelling. As an example, let us mention that for every nice complete graph $K_{n}$, for which $\chi_{\Sigma}\left(K_{n}\right)=3$, there is a proper 3-labelling assigning label 2 only once [2]. Also, for every bipartite graph $G$ with $\chi_{\Sigma}(G)=3$, there exist proper 3-labellings assigning label 3 at most twice [3]. It might be that, in general, using three labels might be too powerful, in the sense that two labels are "almost enough".

Our results in this work are related to a recent take on those questions by Baudon, Pilśniak, Przybyło, Senhaji, Sopena, and Woźniak. In [1], they investigated proper labellings in which all labels must be assigned about the same number of times. More precisely, given a labelling $\ell$ of a graph $G$, one can define, for every label $\alpha$ assigned by $\ell$, the value $\operatorname{nb}_{\ell}(\alpha)$ being the number of edges of $G$ assigned label $\alpha$. We call $\ell$ equitable if, for every two distinct labels $\alpha, \beta$ assigned by $\ell$, the values $\operatorname{nb}_{\ell}(\alpha)$ and $\operatorname{nb}_{\ell}(\beta)$ differ by at most 1 . We denote by $\overline{\chi_{\Sigma}}(G)$ the smallest $k$ such that $G$ admits an equitable proper $k$-labelling. Again, this parameter $\overline{\chi_{\Sigma}}$ is defined for every nice graph.

The authors of [1] have investigated several aspects of equitable proper labellings, most of which are about the relationship between $\chi_{\Sigma}(G)$ and $\overline{\chi_{\Sigma}}(G)$ for a given graph $G$. For a few families of graphs $G$, they have notably established that $\overline{\chi_{\Sigma}}(G)=\chi_{\Sigma}(G)$ holds, except for a few exceptions. In particular:

- For nice forests $F$, we always have $\overline{\chi_{\Sigma}}(F)=\chi_{\Sigma}(F) \leq 2$.

- For nice complete bipartite graphs $K_{n, m}$, we always have $\overline{\chi_{\Sigma}}\left(K_{n, m}\right)=\chi_{\Sigma}\left(K_{n, m}\right) \leq 2$, except for the peculiar case of $K_{3,3}$ which verifies $2=\chi_{\Sigma}\left(K_{3,3}\right)<\overline{\chi_{\Sigma}}\left(K_{3,3}\right)=3$.

- For nice complete graphs $K_{n}$, we always have $\overline{\chi_{\Sigma}}\left(K_{n}\right)=\chi_{\Sigma}\left(K_{n}\right)=3$, except for the peculiar case of $K_{4}$ which verifies $3=\chi_{\Sigma}\left(K_{4}\right)<\overline{\chi_{\Sigma}}\left(K_{4}\right)=4$.

At this point, the previous results lead to a number of interesting questions. Is $K_{4}$ the only graph $G$ with $\overline{\chi_{\Sigma}}(G)>3$ ? Are graphs $G$ with $\chi_{\Sigma}(G)<\overline{\chi_{\Sigma}}(G)$ rare? Can the difference between $\chi_{\Sigma}(G)$ and $\overline{\chi_{\Sigma}}(G)$ be arbitrarily large? In general, is an equitable version of the 1-2-3 Conjecture plausible?

Conjecture 1.2 (Equitable 1-2-3 Conjecture). If $G$ is a connected graph different from $K_{2}$ and $K_{4}$, then $\overline{\chi_{\Sigma}}(G) \leq 3$.

A few more results partially answering some of these questions can be found in Senhaji's thesis [11]. In particular:

- Senhaji proved that $\overline{\chi_{\Sigma}}(G)=\chi_{\Sigma}(G) \leq 3$ holds for a certain number of graphs $G$, including nice paths, nice cycles, some Theta graphs, and some Cartesian products.

- Using computer programs, he came up with four cubic bipartite graphs $G$ verifying $2=$ $\chi_{\Sigma}(G)<\overline{\chi_{\Sigma}}(G)=3$. 
- For particular cubic bipartite graphs $G$, such as Hamiltonian ones, he proved that $\overline{\chi \Sigma}(G) \leq 2$.

In this work, we provide results towards some of the questions above. In particular, we investigate the existence of graphs $G$ with $\chi_{\Sigma}(G)<\overline{\chi_{\Sigma}}(G)$. In Section 2, we first prove that there exist infinitely many such graphs. This is obtained through proving that the problem of deciding whether $\overline{\chi_{\Sigma}}(G)=2$ holds for a given graph $G$ with $\chi_{\Sigma}(G)=2$ is NP-complete. We then investigate, in Section 3, the same question for bipartite graphs. We exhibit operations establishing that there exist infinitely many bipartite graphs $G$ with $\chi_{\Sigma}(G)<\overline{\chi_{\Sigma}}(G)$. We also prove that for every bipartite graph $G$ with $\chi_{\Sigma}(G)=3$, we have $\overline{\chi_{\Sigma}}(G)=3$. In Section 4 , we finally provide a result on equitable proper labellings of regular bipartite graphs, showing that $\overline{\chi_{\Sigma}}(G) \leq k$ holds for every such $k$-regular graph $(k \geq 3)$. In particular, we have $\overline{\chi \Sigma}(G) \leq 3$ for every cubic bipartite graph $G$.

\section{$2 \quad$ NP-completeness result}

This section is devoted to the proof that the problem of deciding whether $\overline{\chi \Sigma}(G)=2$ holds for a given graph $G$ with $\chi_{\Sigma}(G)=2$ is NP-complete.

The reduction in the proof of our main result below will be mostly obtained by plugging several gadgets with specific properties together. More precisely, some of our gadgets will have specific pendent edges (i.e., with exactly one of their ends being of degree 1) being their inputs or outputs. Given two disjoint gadgets $G$ and $H$ where $e$ is an output of $G$ and $f$ is an input of $H$, by plugging $G$ and $H$ (along $e$ and $f$ ) we mean identifying $e$ and $f$ together. More precisely, if $e=x y$ and $f=u v$ with $y$ and $v$ being the vertices of degree 1 of $e$ and $f$ respectively, then identifying $e$ and $f$ means identifying $x$ and $v$, and $y$ and $u$ respectively.

Before proceeding with introducing the needed gadgets, let us first recall the following easy observation that will serve throughout this work.

Observation 2.1. Let $G$ be a graph with a path $(u, v, w, x)$ where $d(v)=d(w)=2$. For any proper labelling $\ell$ of $G$, we have $\ell(u v) \neq \ell(w x)$.

Proof. This is because we would have $c_{\ell}(v)=c_{\ell}(w)$ otherwise.

\subsection{Initiator gadget}

The first gadget we need is the diamond $D$ depicted in Figure 1 . Here and further, for every gadget introduced through a figure, we deal with its vertices and edges following the notation from that figure. The input of $D$ is the edge $u_{1} u_{2}$, while the output of $D$ is the edge $u_{9} u_{10}$. The properties of interest of $D$ are the following:

Theorem 2.2. $D$ verifies the following:

- $|E(D)|=11$.

- In any proper 2-labelling $\ell$ of $D$, we have $\ell\left(u_{1} u_{2}\right)=\ell\left(u_{9} u_{10}\right)$.

- There exist both proper 2-labellings $\ell$ of $D$ where $\ell\left(u_{1} u_{2}\right)=1$, and proper 2-labellings $\ell$ of $D$ where $\ell\left(u_{1} u_{2}\right)=2$.

- In any proper 2 -labelling $\ell$ of $D$ where $\ell\left(u_{1} u_{2}\right)=1$ :

$-c_{\ell}\left(u_{2}\right)=4$

$-c_{\ell}\left(u_{9}\right)$ can be any value in $\{2,3\}$;

$-\mathrm{nb}_{\ell}(1)=7$ and $\mathrm{nb}_{\ell}(2)=4$.

- In any proper 2 -labelling $\ell$ of $D$ where $\ell\left(u_{1} u_{2}\right)=2$ : 


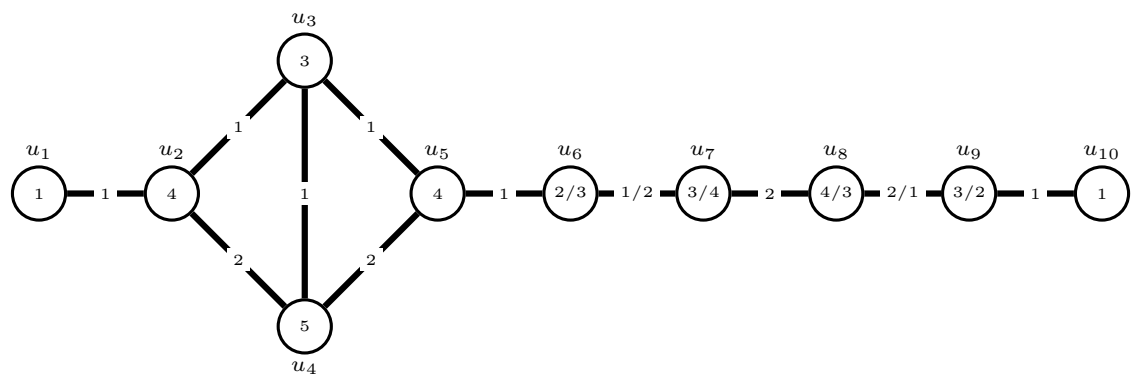

(a) $\ell\left(u_{1} u_{2}\right)=1$.

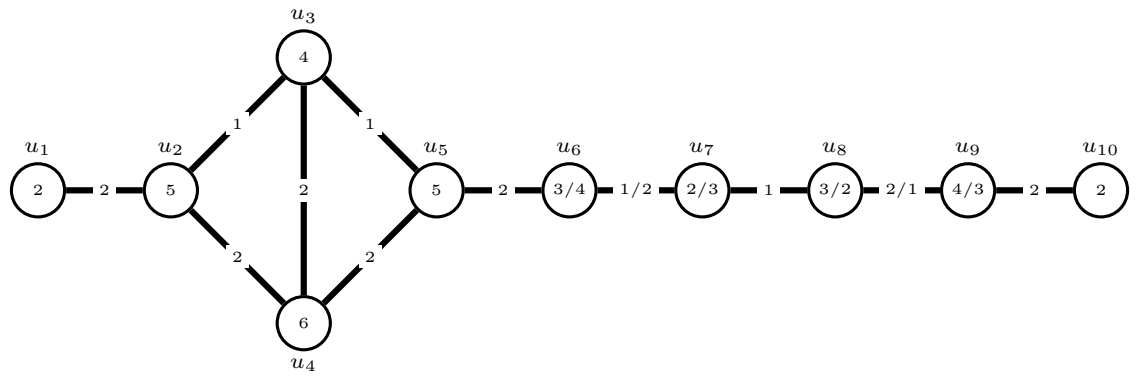

(b) $\ell\left(u_{1} u_{2}\right)=2$.

Figure 1: The diamond gadget $D$. The values in each vertex $v$ are the possible colours of $c_{\ell}(v)$ by a proper 2-labelling $\ell$ of $D$.

$-c_{\ell}\left(u_{2}\right)=5$

- $c_{\ell}\left(u_{9}\right)$ can be any value in $\{3,4\}$;

$-\mathrm{nb}_{\ell}(1)=4$ and $\mathrm{nb}_{\ell}(2)=7$.

Proof. Let $\ell$ be a proper 2-labelling of $D$. Assume $\ell\left(u_{1} u_{2}\right)=1$.

If $\ell\left(u_{2} u_{3}\right)=\ell\left(u_{2} u_{4}\right)=1$, then $c_{\ell}\left(u_{2}\right)=3$. Since $c_{\ell}\left(u_{3}\right) \neq c_{\ell}\left(u_{4}\right)$, we have $\ell\left(u_{3} u_{5}\right)=1$ and $\ell\left(u_{4} u_{5}\right)=2$, or vice versa. Since $c_{\ell}\left(u_{3}\right) \neq c_{\ell}\left(u_{2}\right)$, we have $\ell\left(u_{3} u_{4}\right)=2$. This gives $c_{\ell}\left(u_{3}\right)=4$ and $c_{\ell}\left(u_{4}\right)=5$. Now we note that no matter what $\ell\left(u_{5} u_{6}\right)$ is, we necessarily get $c_{\ell}\left(u_{5}\right) \in\{4,5\}=$ $\left\{c_{\ell}\left(u_{3}\right), c_{\ell}\left(u_{4}\right)\right\}$, a contradiction. So we cannot have $\ell\left(u_{2} u_{3}\right)=\ell\left(u_{2} u_{4}\right)=1$.

If $\ell\left(u_{2} u_{3}\right)=\ell\left(u_{2} u_{4}\right)=2$, then $c_{\ell}\left(u_{2}\right)=5$. Again, since $c_{\ell}\left(u_{3}\right) \neq c_{\ell}\left(u_{4}\right)$, we have, say, $\ell\left(u_{3} u_{5}\right)=$ 1 and $\ell\left(u_{4} u_{5}\right)=2$. Since $c_{\ell}\left(u_{4}\right) \neq 5$, we have $\ell\left(u_{3} u_{4}\right)=2$, which gives $c_{\ell}\left(u_{3}\right)=c_{\ell}\left(u_{2}\right)=5$, a contradiction. Thus, we cannot have $\ell\left(u_{2} u_{3}\right)=\ell\left(u_{2} u_{4}\right)=2$.

If, say, $\ell\left(u_{2} u_{3}\right)=1$ and $\ell\left(u_{2} u_{4}\right)=2$, then $c_{\ell}\left(u_{2}\right)=4$. Assume first that $\ell\left(u_{3} u_{4}\right)=2$. In that case, since $c_{\ell}\left(u_{3}\right) \neq c_{\ell}\left(u_{2}\right)$, we have $\ell\left(u_{3} u_{5}\right)=2$, and thus $c_{\ell}\left(u_{3}\right)=5$. Since $c_{\ell}\left(u_{4}\right) \neq c_{\ell}\left(u_{3}\right)$, we have $\ell\left(u_{4} u_{5}\right)=2$, and thus $c_{\ell}\left(u_{4}\right)=6$. Now, we note that no matter what $\ell\left(u_{5} u_{6}\right)$ is, we have $c_{\ell}\left(u_{5}\right) \in\{5,6\}=\left\{c_{\ell}\left(u_{3}\right), c_{\ell}\left(u_{4}\right)\right\}$, a contradiction. So, we have $\ell\left(u_{3} u_{4}\right)=1$. Since $c_{\ell}\left(u_{3}\right)$ and $c_{\ell}\left(u_{4}\right)$ are different from $c_{\ell}\left(u_{2}\right)$, we have $\ell\left(u_{3} u_{5}\right)=1$ and $\ell\left(u_{4} u_{5}\right)=2$, which gives $c_{\ell}\left(u_{3}\right)=3$ and $c_{\ell}\left(u_{4}\right)=5$. Now, since $c_{\ell}\left(u_{5}\right) \notin\{3,5\}=\left\{c_{\ell}\left(u_{3}\right), c_{\ell}\left(u_{4}\right)\right\}$, we have $\ell\left(u_{5} u_{6}\right)=1$, which gives $c_{\ell}\left(u_{5}\right)=4$. By Observation 2.1, we then have $\ell\left(u_{7} u_{8}\right) \neq \ell\left(u_{5} u_{6}\right)$ and $\ell\left(u_{9} u_{10}\right) \neq \ell\left(u_{7} u_{8}\right)$, and thus $\ell\left(u_{7} u_{8}\right)=2$ and $\ell\left(u_{9} u_{10}\right)=1$. By the same argument, we have $\ell\left(u_{6} u_{7}\right) \neq \ell\left(u_{8} u_{9}\right)$, and both ways are possible. Indeed, if on the one hand $\ell\left(u_{6} u_{7}\right)=1$ and $\ell\left(u_{8} u_{9}\right)=2$, then $c_{\ell}\left(u_{6}\right)=2, c_{\ell}\left(u_{7}\right)=3$, $c_{\ell}\left(u_{8}\right)=4$, and $c_{\ell}\left(u_{9}\right)=3$. If on the other hand $\ell\left(u_{6} u_{7}\right)=2$ and $\ell\left(u_{8} u_{9}\right)=1$, then $c_{\ell}\left(u_{6}\right)=3$, $c_{\ell}\left(u_{7}\right)=4, c_{\ell}\left(u_{8}\right)=3$, and $c_{\ell}\left(u_{9}\right)=2$. According to all these arguments, we have that $\operatorname{nb}_{\ell}(1)=7$ while $\operatorname{nb}_{\ell}(2)=4$. Also, depending on whether $\ell\left(u_{6} u_{7}\right)=1$ and $\ell\left(u_{8} u_{9}\right)=2$, or $\ell\left(u_{6} u_{7}\right)=2$ and 
$\ell\left(u_{8} u_{9}\right)=1$, the value of $c_{\ell}\left(u_{9}\right)$ can be any one in $\{2,3\}$. See Figure 1 (a) for an illustration of the resulting $\ell$.

These arguments can be mimicked the exact same way when $\ell\left(u_{1} u_{2}\right)=2$. In particular, we have $\ell\left(u_{1} u_{2}\right)=\ell\left(u_{9} u_{10}\right)=2, \operatorname{nb}_{\ell}(1)=4$ while $\operatorname{nb}_{\ell}(2)=7$, and $c_{\ell}\left(u_{9}\right)$ can be any value in $\{3,4\}$. See Figure 1(b) for an illustration.

The initiator gadget $I_{k}$ of length $k \geq 2$ has one input and one output, and is obtained from $k$ diamond gadgets as follows. For $k=2$, the initiator gadget $I_{2}$ of length 2 is obtained by plugging two copies $D_{1}$ and $D_{2}$ of the diamond gadget $D$ along the output of $D_{1}$ and the input of $D_{2}$. The input of $I_{2}$ is then the input of $D_{1}$ and the output of $I_{2}$ is then the output of $D_{2}$. For $k>2$, the initiator gadget $I_{k}$ of length $k$ is obtained by plugging a copy $G$ of the initiator gadget $I_{k-1}$ of length $k-1$ and a new copy $H$ of the diamond gadget $D$ along the output of $G$ and the input of $H$. The input of $I_{k}$ is then the input of $G$ and the output of $I_{k}$ is then the output of $H$.

Theorem 2.3. $I_{k}$ verifies the following, for every $k \geq 2$ :

- $\left|E\left(I_{k}\right)\right|=10 k+1$.

- In any proper 2-labelling of $I_{k}$, the input and output are assigned the same label.

- There exist both proper 2-labellings of $I_{k}$ where the input is assigned label 1 , and proper 2labellings of $I_{k}$ where the input is assigned label 2.

- In any proper 2-labelling $\ell$ of $I_{k}$ where the input is assigned label 1:

$-c_{\ell}(v)$ can be any value in $\{2,3\}$, where $v$ denotes the degree-2 vertex of the output of $I_{k}$;

$-\mathrm{nb}_{\ell}(1)=6 k+1$ and $\mathrm{nb}_{\ell}(2)=4 k$.

- In any proper 2-labelling $\ell$ of $I_{k}$ where the input is assigned label 2:

$-c_{\ell}(v)$ can be any value in $\{3,4\}$, where $v$ denotes the degree-2 vertex of the output of $I_{k}$;

$-\mathrm{nb}_{\ell}(1)=4 k$ and $\mathrm{nb}_{\ell}(2)=6 k+1$.

Proof. This follows mainly from the fact that $I_{k}$ is made up of $k$ copies of the diamond gadget $D$ plugged one after another, and that the diamond gadget $D$ has all of the properties described in Theorem 2.2. In particular, a proper 2-labelling of $I_{k}$ induces a proper 2-labelling of the $k$ copies of the diamond gadget $D$ in it. Specifically, it can be checked that no conflict can arise around the inputs and outputs that were identified. Also, for a proper 2-labelling of $I_{k}$ assigning label $\alpha \in\{1,2\}$ to the input, in each copy of the diamond gadget $D$ label $\alpha$ must be assigned to 7 edges while the other label must be assigned to 4 edges. Due to how the copies of $D$ were plugged, we deduce that $7 k-(k-1)=6 k+1$ edges of $I_{k}$ must be assigned label $\alpha$, while $4 k$ edges must be assigned the other label.

\subsection{Corrector gadget}

The corrector gadget $C$ is the graph depicted in Figure 2. The input of $C$ is the edge $u_{1} u_{2}$, while $C$ has no output. Its interesting properties are the following:

Theorem 2.4. $C$ verifies the following:

- $|E(C)|=9$.

- There exist both proper 2-labellings $\ell$ of $C$ where $\ell\left(u_{1} u_{2}\right)=1$, and proper 2-labellings $\ell$ of $C$ where $\ell\left(u_{1} u_{2}\right)=2$.

- In any proper 2 -labelling $\ell$ of $C$ where $\ell\left(u_{1} u_{2}\right)=1$ : 


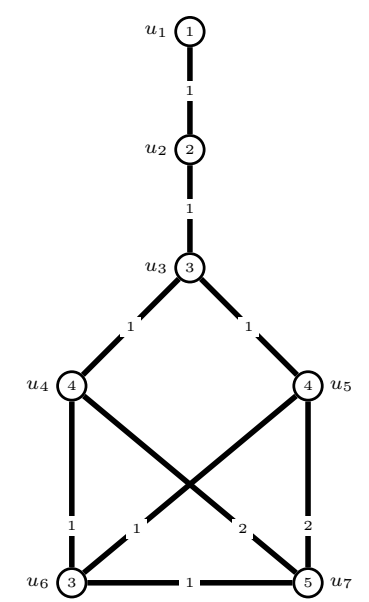

(a) $\ell\left(u_{1} u_{2}\right)=1, \operatorname{nb}_{\ell}(1)=7$, $\mathrm{nb}_{\ell}(2)=2$.

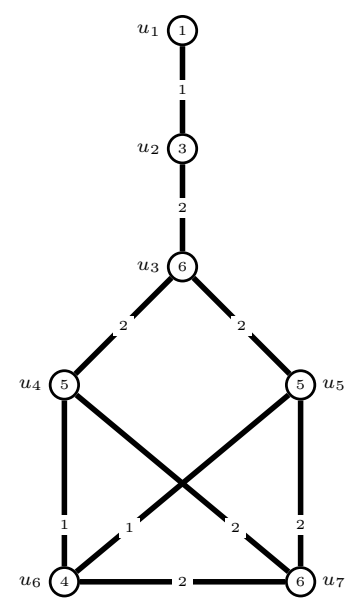

(b) $\ell\left(u_{1} u_{2}\right)=1, \mathrm{nb}_{\ell}(1)=3$, $\mathrm{nb}_{\ell}(2)=6$.

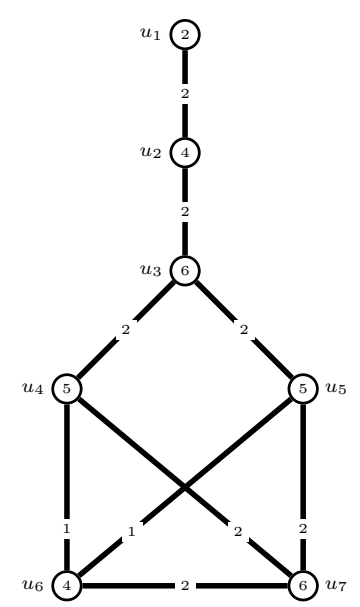

(c) $\ell\left(u_{1} u_{2}\right)=1, \operatorname{nb}_{\ell}(1)=2$, $\operatorname{nb}_{\ell}(2)=7$.

Figure 2: The corrector gadget $C$. The values in each vertex $v$ are the possible colours of $c_{\ell}(v)$ by a proper 2-labelling $\ell$ of $C$.

$-c_{\ell}\left(u_{2}\right) \in\{2,3\}$

- either $\mathrm{nb}_{\ell}(1)=7$ and $\mathrm{nb}_{\ell}(2)=2$, or $\mathrm{nb}_{\ell}(1)=3$ and $\mathrm{nb}_{\ell}(2)=6$.

- In any proper 2 -labelling $\ell$ of $C$ where $\ell\left(u_{1} u_{2}\right)=2$ :

$-c_{\ell}\left(u_{2}\right)=4$

$-\mathrm{nb}_{\ell}(1)=2$ and $\mathrm{nb}_{\ell}(2)=7$.

Proof. Let $\ell$ be a proper 2-labelling of $C$. Because $u_{6}$ and $u_{7}$ both have degree 3 , we have that $c_{\ell}\left(u_{6}\right), c_{\ell}\left(u_{7}\right) \in\{3,4,5,6\}$. Furthermore, $c_{\ell}\left(u_{6}\right) \neq c_{\ell}\left(u_{7}\right)$, and we cannot have $\left\{c_{\ell}\left(u_{6}\right), c_{\ell}\left(u_{7}\right)\right\}=$ $\{3,6\}$. We consider all of the remaining possibilities for $\left\{c_{\ell}\left(u_{6}\right), c_{\ell}\left(u_{7}\right)\right\}$ in what follows.

Assume $c_{\ell}\left(u_{6}\right)=3$ and $c_{\ell}\left(u_{7}\right)=4$. Then, all three edges incident to $u_{6}$ must be labelled 1 , while we have, say, $\ell\left(u_{7} u_{4}\right)=1$ while $\ell\left(u_{7} u_{5}\right)=2$. Then, we note that, whatever $\ell\left(u_{4} u_{3}\right)$ is, we have $c_{\ell}\left(u_{4}\right) \in\{3,4\}=\left\{c_{\ell}\left(u_{6}\right), c_{\ell}\left(u_{7}\right)\right\}$, a contradiction. The case where $\ell\left(u_{7} u_{4}\right)=2$ while $\ell\left(u_{7} u_{5}\right)=1$ is symmetric with the colour of $u_{5}$ coming into conflict with that of $u_{6}$ or $u_{7}$ instead. Thus, we cannot have $\left\{c_{\ell}\left(u_{6}\right), c_{\ell}\left(u_{7}\right)\right\}=\{3,4\}$.

Assume $c_{\ell}\left(u_{6}\right)=3$ and $c_{\ell}\left(u_{7}\right)=5$. Again, all three edges incident to $u_{6}$ must be labelled 1 , while we have $\ell\left(u_{7} u_{4}\right)=\ell\left(u_{7} u_{5}\right)=2$. Now, since $c_{\ell}\left(u_{4}\right)$ and $c_{\ell}\left(u_{5}\right)$ are different from $5=c_{\ell}\left(u_{7}\right)$, we have $\ell\left(u_{4} u_{3}\right)=\ell\left(u_{5} u_{3}\right)=1$. This gives $c_{\ell}\left(u_{4}\right)=c_{\ell}\left(u_{5}\right)=4$. Now, since $c_{\ell}\left(u_{3}\right)$ is different from $4=c_{\ell}\left(u_{4}\right)=c_{\ell}\left(u_{5}\right)$, we have $\ell\left(u_{3} u_{2}\right)=1$. Then $c_{\ell}\left(u_{3}\right)=3$, and, since $c_{\ell}\left(u_{2}\right) \neq c_{\ell}\left(u_{3}\right)$, we have $\ell\left(u_{2} u_{1}\right)=1$, which yields $c_{\ell}\left(u_{2}\right)=2$. This is the labelling depicted in Figure 2(a).

Assume $c_{\ell}\left(u_{6}\right)=4$ and $c_{\ell}\left(u_{7}\right)=5$. First, assume $\ell\left(u_{6} u_{7}\right)=1$. Then we have, say, $\ell\left(u_{6} u_{4}\right)=1$ and $\ell\left(u_{6} u_{5}\right)=\ell\left(u_{7} u_{4}\right)=\ell\left(u_{7} u_{5}\right)=2$ (the case where $\ell\left(u_{6} u_{4}\right)=2$ and $\ell\left(u_{6} u_{5}\right)=1$ is symmetric). Note now that whatever $\ell\left(u_{4} u_{3}\right)$ is, we have $c_{\ell}\left(u_{4}\right) \in\{4,5\}=\left\{c_{\ell}\left(u_{6}\right), c_{\ell}\left(u_{7}\right)\right\}$, a contradiction. Then, assume $\ell\left(u_{6} u_{7}\right)=2$. Then we have $\ell\left(u_{6} u_{4}\right)=\ell\left(u_{6} u_{5}\right)=1$ and, say, $\ell\left(u_{7} u_{5}\right)=1$ and $\ell\left(u_{7} u_{4}\right)=2$ (the case where $\ell\left(u_{7} u_{5}\right)=2$ and $\ell\left(u_{7} u_{4}\right)=1$ is symmetric). Again, note that whatever $\ell\left(u_{4} u_{3}\right)$ is, we have $c_{\ell}\left(u_{4}\right) \in\{4,5\}=\left\{c_{\ell}\left(u_{6}\right), c_{\ell}\left(u_{7}\right)\right\}$, a contradiction. Thus, we cannot have $\left\{c_{\ell}\left(u_{6}\right), c_{\ell}\left(u_{7}\right)\right\}=\{4,5\}$

Assume $c_{\ell}\left(u_{6}\right)=4$ and $c_{\ell}\left(u_{7}\right)=6$. Then, all three edges incident to $u_{7}$ must be labelled 2, while we have $\ell\left(u_{6} u_{4}\right)=\ell\left(u_{6} u_{5}\right)=1$. Now, since $c_{\ell}\left(u_{4}\right)$ and $c_{\ell}\left(u_{5}\right)$ are different from $4=c_{\ell}\left(u_{6}\right)$, we have $\ell\left(u_{4} u_{3}\right)=\ell\left(u_{5} u_{3}\right)=2$. This gives $c_{\ell}\left(u_{4}\right)=c_{\ell}\left(u_{5}\right)=5$. Now, since $c_{\ell}\left(u_{3}\right)$ is different from 


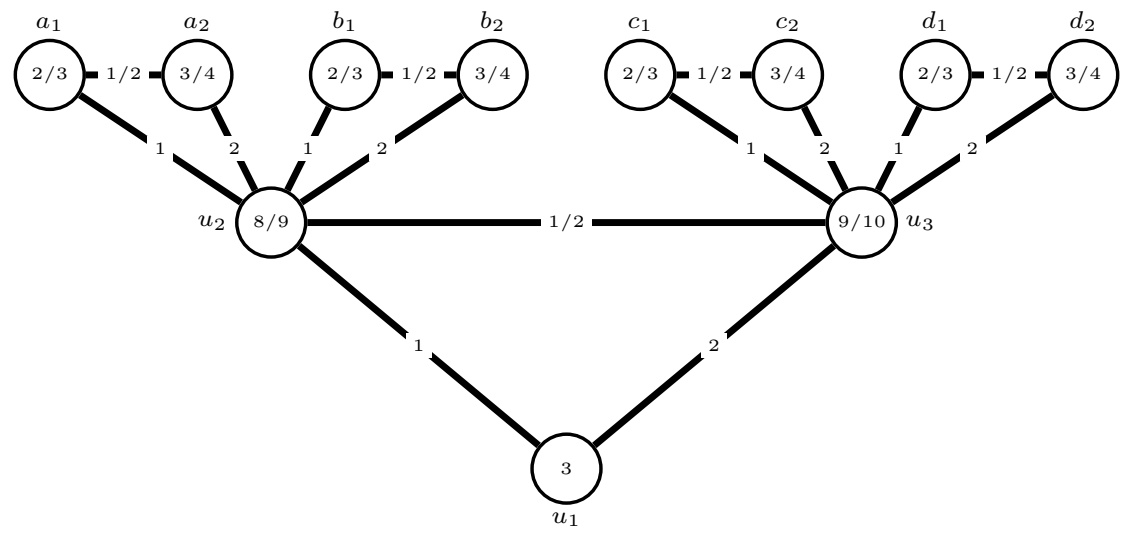

Figure 3: The triangle gadget $T_{2}$. The values in each vertex $v$ are the possible colours of $c_{\ell}(v)$ by a proper 2-labelling $\ell$ of $T_{2}$.

$5=c_{\ell}\left(u_{4}\right)=c_{\ell}\left(u_{5}\right)$, we have $\ell\left(u_{3} u_{2}\right)=2$. Then, $c_{\ell}\left(u_{3}\right)=6$, and note that $u_{1} u_{2}$ can correctly be assigned either of the labels 1 and 2. In the first case, we get the labelling depicted in Figure 2(b), in which $c_{\ell}\left(u_{2}\right)=3$. In the second case, we get the labelling depicted in Figure 2(c), in which $c_{\ell}\left(u_{2}\right)=4$.

Assume $c_{\ell}\left(u_{6}\right)=5$ and $c_{\ell}\left(u_{7}\right)=6$. Then, all three edges incident to $u_{7}$ must be labelled 2, while we have, say, $\ell\left(u_{6} u_{4}\right)=1$ while $\ell\left(u_{6} u_{5}\right)=2$ (the case where $\ell\left(u_{6} u_{4}\right)=2$ while $\ell\left(u_{6} u_{5}\right)=1$ is symmetric). Then, we note that, whatever $\ell\left(u_{5} u_{3}\right)$ is, we have $c_{\ell}\left(u_{5}\right) \in\{5,6\}=\left\{c_{\ell}\left(u_{6}\right), c_{\ell}\left(u_{7}\right)\right\}$, a contradiction. Thus, we cannot have $\left\{c_{\ell}\left(u_{6}\right), c_{\ell}\left(u_{7}\right)\right\}=\{5,6\}$.

\subsection{Generator gadget}

Let $T_{2}$ be the triangle gadget depicted in Figure 3, which has no input nor output. We call $u_{1}$ the root of $T_{2}$. Note that $T_{2}$ has 15 edges. It has the following labelling properties:

Theorem 2.5. In any proper 2-labelling $\ell$ of $T_{2}$ :

- $\left\{\ell\left(u_{1} u_{2}\right), \ell\left(u_{1} u_{3}\right)\right\}=\{1,2\}$;

- $\left\{c_{\ell}\left(u_{2}\right), c_{\ell}\left(u_{3}\right)\right\}=\{8,9\}$ or $\left\{c_{\ell}\left(u_{2}\right), c_{\ell}\left(u_{3}\right)\right\}=\{9,10\}$; furthermore:

- if $\left\{c_{\ell}\left(u_{2}\right), c_{\ell}\left(u_{3}\right)\right\}=\{8,9\}$, then $\mathrm{nb}_{\ell}(1)$ can be any value in $\{6, \ldots, 10\}$;

- if $\left\{c_{\ell}\left(u_{2}\right), c_{\ell}\left(u_{3}\right)\right\}=\{9,10\}$, then $\mathrm{nb}_{\ell}(1)$ can be any value in $\{5, \ldots, 9\}$.

Proof. Let $\ell$ be a proper 2-labelling of $T_{2}$. Since $c_{\ell}\left(a_{1}\right) \neq c_{\ell}\left(a_{2}\right)$, we have, say, $\ell\left(a_{1} u_{2}\right)=1$ and $\ell\left(a_{2} u_{2}\right)=2$. Note that whatever $\ell\left(a_{1} a_{2}\right)$ is, no conflict involving $a_{1}$ (or $a_{2}$ ) and $u_{2}$ can arise, due to the larger degree of $u_{2}$. These arguments also apply around the $b_{i}$ 's, $c_{i}$ 's, and $d_{i}$ 's. In particular, the labels of the four edges joining $u_{2}$ to the $a_{i}$ 's and $b_{i}$ 's bring 6 to the colour of $u_{2}$, and similarly the labels of the four edges joining $u_{3}$ to the $c_{i}$ 's and $d_{i}$ 's bring 6 to the colour of $u_{3}$. Now, since $c_{\ell}\left(u_{2}\right) \neq c_{\ell}\left(u_{3}\right)$, we have, say, $\ell\left(u_{1} u_{2}\right)=1$ and $\ell\left(u_{1} u_{3}\right)=2$. Then, no conflict involving $u_{2}$ and $u_{3}$ can arise, no matter whether $u_{2} u_{3}$ is labelled 1 or 2 . In the first case, we get $\left(c_{\ell}\left(u_{2}\right), c_{\ell}\left(u_{3}\right)\right)=(8,9)$, while we get $\left(c_{\ell}\left(u_{2}\right), c_{\ell}\left(u_{3}\right)\right)=(9,10)$ in the second case.

The parts of the statement dealing with $\mathrm{nb}_{\ell}(1)$ hold, essentially, because each of the edges $a_{1} a_{2}$, $b_{1} b_{2}, c_{1} c_{2}, d_{1} d_{2}$, and $u_{2} u_{3}$ can freely be assigned any label in $\{1,2\}$ by $\ell$. 


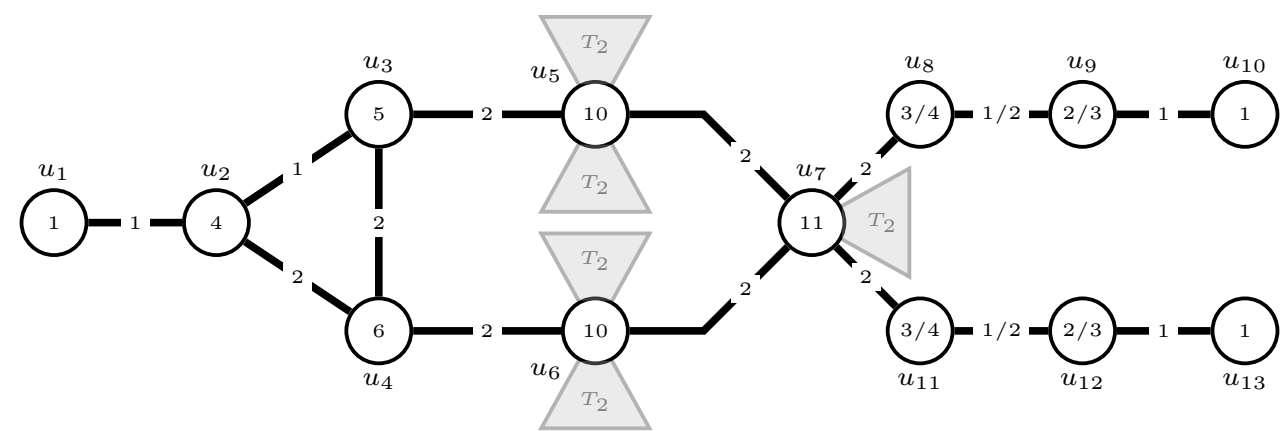

(a) $\ell\left(u_{1} u_{2}\right)=1$.

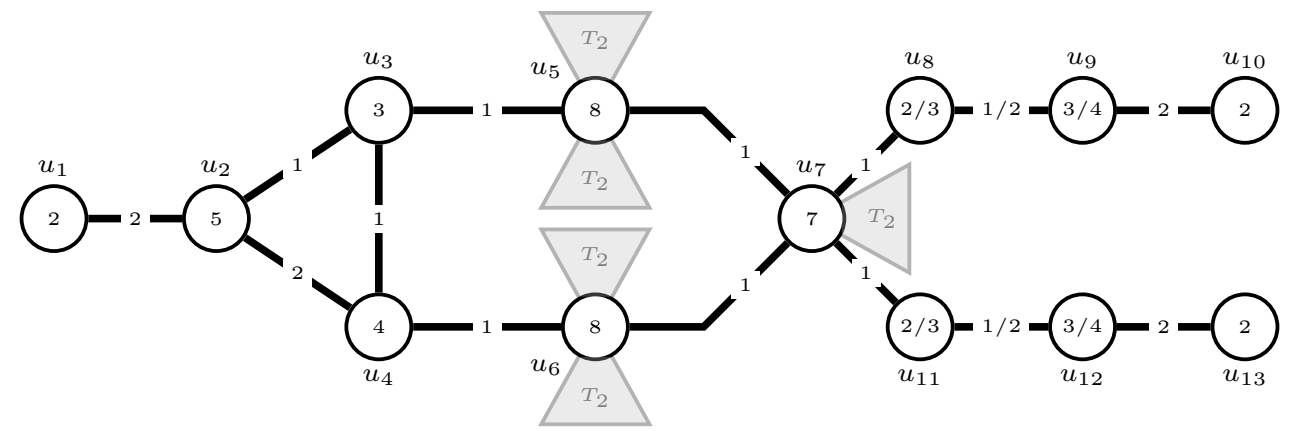

(b) $\ell\left(u_{1} u_{2}\right)=2$.

Figure 4: The spreading gadget $G^{\curlywedge}$. Every triangle with a " $T_{2}$ " inside indicates that a copy of the triangle gadget $T_{2}$ is attached via its root. The values in each vertex $v$ are the possible colours of $c_{\ell}(v)$ by a proper 2-labelling $\ell$ of $G^{\curlywedge}$.

The spreading gadget $G^{\curlywedge}$ is depicted in Figure 4. The input of $G^{\curlywedge}$ is its edge $u_{1} u_{2}$, while its two outputs are its edges $u_{9} u_{10}$ and $u_{12} u_{13}$. Note that $G^{\curlywedge}$ contains five copies of the triangle gadget $T_{2}$ attached via their roots. The properties of interest of $G^{\curlywedge}$ are the following:

Theorem 2.6. $G^{\curlywedge}$ verifies the following:

- $\left|E\left(G^{\curlywedge}\right)\right|=89$.

- In any proper 2-labelling $\ell$ of $G^{\curlywedge}$, we have $\ell\left(u_{1} u_{2}\right)=\ell\left(u_{9} u_{10}\right)=\ell\left(u_{12} u_{13}\right)$.

- There exist both proper 2-labellings $\ell$ of $G^{\curlywedge}$ where $\ell\left(u_{1} u_{2}\right)=1$, and proper 2-labellings $\ell$ of $G^{\curlywedge}$ where $\ell\left(u_{1} u_{2}\right)=2$.

- In any proper 2-labelling $\ell$ of $G^{\curlywedge}$ where $\ell\left(u_{1} u_{2}\right)=1$ :

$-c_{\ell}\left(u_{2}\right)=4$

- $c_{\ell}\left(u_{9}\right)$ and $c_{\ell}\left(u_{12}\right)$ can be any value in $\{2,3\}$; furthermore:

* if $c_{\ell}\left(u_{9}\right)=c_{\ell}\left(u_{12}\right)=2$, then $\mathrm{nb}_{\ell}(1)$ can be any value in $\{35, \ldots, 56\}$;

* if $c_{\ell}\left(u_{9}\right)=c_{\ell}\left(u_{12}\right)=3$, then $\mathrm{nb}_{\ell}(1)$ can be any value in $\{33, \ldots, 54\}$;

* if $\left\{c_{\ell}\left(u_{9}\right), c_{\ell}\left(u_{12}\right)\right\}=\{2,3\}$, then $\mathrm{nb}_{\ell}(1)$ can be any value in $\{34, \ldots, 55\}$;

- In any proper 2-labelling $\ell$ of $G^{\curlywedge}$ where $\ell\left(u_{1} u_{2}\right)=2$ :

$-c_{\ell}\left(u_{2}\right)=5$

- $c_{\ell}\left(u_{9}\right)$ and $c_{\ell}\left(u_{12}\right)$ can be any value in $\{3,4\}$; furthermore:

* if $c_{\ell}\left(u_{9}\right)=c_{\ell}\left(u_{12}\right)=3$, then $\operatorname{nb}_{\ell}(1)$ can be any value in $\{35, \ldots, 56\}$; 
* if $c_{\ell}\left(u_{9}\right)=c_{\ell}\left(u_{12}\right)=4$, then $\mathrm{nb}_{\ell}(1)$ can be any value in $\{33, \ldots, 54\}$;

* if $\left\{c_{\ell}\left(u_{9}\right), c_{\ell}\left(u_{12}\right)\right\}=\{3,4\}$, then $\mathrm{nb}_{\ell}(1)$ can be any value in $\{34, \ldots, 55\}$.

Proof. Consider $\ell$ a proper 2-labelling of $G^{\curlywedge}$. We first note that we have $\ell\left(u_{3} u_{5}\right)=\ell\left(u_{4} u_{6}\right)$. Indeed, suppose to the contrary that, e.g., $\ell\left(u_{3} u_{5}\right)=1$ and $\ell\left(u_{4} u_{6}\right)=2$ holds. Since there are two copies of $T_{2}$ attached to $u_{5}$, by Theorem 2.5 , the colour of $u_{5}$ is $7+\ell\left(u_{5} u_{7}\right)$ and it is adjacent to a vertex with colour 9 (in $T_{2}$ ). Similarly, because of the two copies of $T_{2}$ attached to $u_{6}$, the colour of $u_{6}$ is $8+\ell\left(u_{6} u_{7}\right)$ and it is adjacent to a vertex with colour 9 (in $\left.T_{2}\right)$. Then, we must have $\ell\left(u_{5} u_{7}\right)=1$ and $\ell\left(u_{6} u_{7}\right)=2$, so that $c_{\ell}\left(u_{5}\right)=8$ and $c_{\ell}\left(u_{6}\right)=10$. We also know that a neighbour of $u_{7}$ from the graph $T_{2}$ attached to it has colour 9 , and that this graph $T_{2}$ provides 3 to the colour of $u_{7}$ by Theorem 2.5. Then, $u_{7}$ has colour $6+\ell\left(u_{7} u_{8}\right)+\ell\left(u_{7} u_{11}\right)$, and the two edges $u_{7} u_{8}$ and $u_{7} u_{11}$ must be labelled (with 1 or 2 ) in such a way that the colour of $u_{7}$ does not meet any value in $\{8,9,10\}$, which is impossible.

Now suppose $\ell\left(u_{1} u_{2}\right)=1$, and consider the edges $u_{2} u_{3}$ and $u_{2} u_{4}$ (see Figure 4(a) for an illustration). First, if $\ell\left(u_{2} u_{3}\right)=\ell\left(u_{2} u_{4}\right)$, then note that $\ell$ cannot be proper according to the arguments above since we would need to have $\ell\left(u_{3} u_{5}\right) \neq \ell\left(u_{4} u_{6}\right)$ since $c_{\ell}\left(u_{3}\right) \neq c_{\ell}\left(u_{4}\right)$. Thus, $\ell\left(u_{2} u_{3}\right)=1$ and $\ell\left(u_{2} u_{4}\right)=2$ without loss of generality, and $c_{\ell}\left(u_{2}\right)=4$. Note that, if $\ell\left(u_{3} u_{4}\right)=1$, then we necessarily get that $c_{\ell}\left(u_{3}\right)$ or $c_{\ell}\left(u_{4}\right)$ is equal to $c_{\ell}\left(u_{2}\right)$ since we need $\ell\left(u_{3} u_{5}\right)=\ell\left(u_{4} u_{6}\right)$. Thus, $\ell\left(u_{3} u_{4}\right)=2$. We then have $\ell\left(u_{3} u_{5}\right)=2$ since $c_{\ell}\left(u_{3}\right) \neq c_{\ell}\left(u_{2}\right)$, and also $\ell\left(u_{4} u_{6}\right)=2$ since $c_{\ell}\left(u_{4}\right) \neq c_{\ell}\left(u_{3}\right)$ (and because $\ell\left(u_{4} u_{6}\right)=\ell\left(u_{3} u_{5}\right)$ by the arguments above).

According to the arguments above, we have $\ell\left(u_{5} u_{7}\right)=\ell\left(u_{6} u_{7}\right)=2$. By the same arguments and since $c_{\ell}\left(u_{5}\right)=c_{\ell}\left(u_{6}\right)=10$, we have $\ell\left(u_{7} u_{8}\right)=\ell\left(u_{7} u_{11}\right)=2$. Then, $\ell\left(u_{9} u_{10}\right)=\ell\left(u_{12} u_{13}\right)=1$ to avoid conflicts. Thus, assuming the input of $G^{\curlywedge}$ is labelled 1 , then its two outputs are also labelled 1. A similar case analysis yields an analogous conclusion when $\ell\left(u_{1} u_{2}\right)=2$, see Figure 4(b).

Let us conclude by pointing out that, in the proper labellings of $G^{\curlywedge}$ mentioned above, the only edges for which the assigned label can freely be either 1 or 2 are the edges $u_{8} u_{9}, u_{11} u_{12}, 4$ edges in each of the four copies of $T_{2}$ attached to $u_{5}$ and $u_{6}$, and 5 edges in the copy of $T_{2}$ attached to $u_{7}$. As pointed out earlier, all other edges must (up to symmetry) receive a particular label in $\{1,2\}$ as soon as that of $u_{1} u_{2}$ is fixed. It is then easy to check that the parts of the statement dealing with $\operatorname{nb}_{\ell}(1)$ are true.

The last gadget we need is the generator gadget $G_{m}$ with $m \geq 3$ outputs and one input, which is obtained from $m-1$ spreading gadgets as follows. For $m=3$, the generator gadget $G_{3}$ with 3 outputs is obtained by plugging two copies $H_{1}$ and $H_{2}$ of the spreading gadget $G^{\curlywedge}$ along any output of $H_{1}$ and the input of $H_{2}$. The input of $G_{3}$ is then the input of $H_{1}$ and the 3 outputs of $G_{3}$ are the second (unplugged) output of $H_{1}$ and the two outputs of $H_{2}$. For $m>3$, the generator gadget $G_{m}$ with $m$ outputs is obtained by plugging a copy $G$ of the generator gadget $G_{m-1}$ with $m-1$ outputs and a new copy $H$ of the spreading gadget $G^{\curlywedge}$ along one output of $G$ and the input of $H$. The input of $G_{m}$ is then the input of $G$ and the $m$ outputs of $G_{m}$ are the remaining $m-2$ (unplugged) outputs of $G$ and the two outputs of $H$.

Theorem 2.7. $G_{m}$ verifies the following, for every $m \geq 3$ :

- $\left|E\left(G_{m}\right)\right|=88 m-87$.

- In any proper 2-labelling of $G_{m}$, the input and $m$ outputs are assigned the same label.

- There exist both proper 2-labellings of $G_{m}$ where the input is assigned label 1 , and proper 2-labellings of $G_{m}$ where the input is assigned label 2.

- In any proper 2 -labelling $\ell$ of $G_{m}$ assigning label 1 to the input, $\mathrm{nb}_{\ell}(1) \in\{32 m-31, \ldots, 55 m-$ $54\}$.

- In any proper 2 -labelling $\ell$ of $G_{m}$ assigning label 2 to the input, $\mathrm{nb}_{\ell}(1) \in\{33 m-33, \ldots, 56 m-$ $56\}$. 
Proof. This follows essentially from Theorem 2.6, since $G_{m}$ is made up of $m-1$ copies of $G^{\curlywedge}$. In particular, any proper 2-labelling $\ell$ of $G_{m}$ induces one of each of its $m-1$ underlying $G^{\curlywedge}$ 's. As pointed out in the statement of Theorem 2.6, vertices identified through the plugging operation cannot get in conflict.

The part of the statement dealing with $\mathrm{nb}_{\ell}(1)$ is essentially because, in each copy of $G^{\curlywedge}$ in $G_{m}$, there are 23 edges that can freely be set to 1 or 2 (4 edges in four attached copies of $T_{2}, 5$ edges in the last attached copy of $T_{2}$, and 2 edges adjacent to the outputs). Assuming the input of $G_{m}$ is labelled 1 by $\ell$, according to Theorem 2.6 in each of the copies of $G^{\curlywedge}$ the number of edges that can be assigned label 1 essentially ranges from 33 to 56 . Thus, in $G_{m}$, the number of edges that can be assigned label 1 ranges from $33(m-1)-(m-2)=32 m-31$ to $56(m-1)-(m-2)=55 m-54$. The computation is similar when the input of $G_{m}$ is assigned label 2, the only difference is that copies of $G^{\curlywedge}$ do not share edges labelled 1.

\subsection{Main result}

We are now ready for the main result of this section.

Theorem 2.8. Given a graph $G$ with $\chi_{\Sigma}(G)=2$, deciding if $\overline{\chi_{\Sigma}}(G)=\chi_{\Sigma}(G)$ is NP-complete.

Proof. The problem is clearly in NP, so we focus on proving it is NP-hard. We do it by reduction from the Monotone Cubic 1-IN-3 SAT problem, which is NP-hard according to [8]. An instance of this problem is a $3 \mathrm{CNF}$ formula $F$ in which every clause $C_{j}=\left(x_{1} \vee x_{2} \vee x_{3}\right)$ contains exactly three distinct variables (not negated) and every variable $x_{i}$ belongs to exactly three distinct clauses. The question is whether there is a 1-in-3 truth assignment to the variables of $F$, i.e., a truth assignment such that every clause has exactly one true variable. Given $F$, we construct, in polynomial time, a graph $G$ such that $F$ admits a 1-in-3 truth assignment $\phi$ if and only if $G$ admits an equitable proper 2-labelling $\ell$.

High-level description. Before describing the explicit construction of $G$, let us first give some intuition about its desired structure, and how all the previous gadgets will be used.

The most important gadget for our construction is the generator gadget $G_{m}$, as the fact that we can generate arbitrarily many pending edges with the same label by a proper 2-labelling is a very convenient feature. This, for instance, permits to make the colours of some vertices grow by a similar amount, or, as will be illustrated later, to forbid some values as vertex colours.

$G_{m}$ has several downsides, however. A first one is that we, a priori, do not know whether its input and outputs will be labelled 1 or 2 by a proper 2-labelling. A second one is that $G_{m}$ does not comply well with equitability, in the sense that, generally speaking, it admits both proper 2-labellings highly favouring the number of assigned 1's, and proper 2-labellings highly favouring the number of assigned 2's.

To overcome these points, we will use the initiator gadget $I_{k}$ and copies of the corrector gadget $C$, in the following way. The initiator gadget $I_{k}$ will be used to introduce a large imbalance in favour of one of the two labels by any proper 2-labelling. By that, we mean an imbalance that is so big that even all the labelling freedom we have in $G_{m}$ will not allow to close the gap. To make sure that the whole graph does admit equitable proper 2-labellings, however, we will add several copies of the corrector gadget $C$. The most important property of this gadget is that, in terms of equitability, its behaviour regarding label 1 and label 2 is far from symmetric. By that, we mean, as noted in Theorem 2.4, that the possibilities $C$ grants highly depend on the label assigned to its input by a proper 2-labelling. If this label is 1, then we can both favour the number of assigned 1's or favour the number of assigned 2's. On the contrary, if this label is 2, then for sure the number of assigned 2's is favoured. 


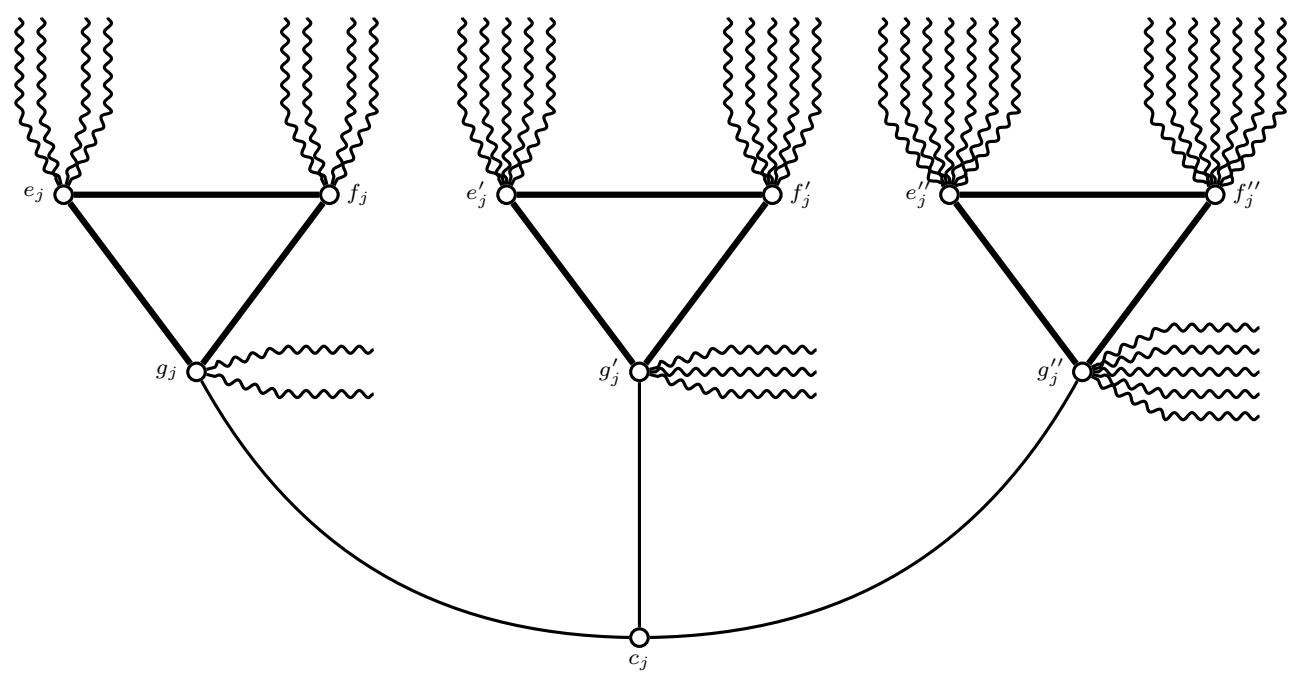

Figure 5: Structure around a clause vertex $c_{j}$. Wiggly edges are outputs of $G_{\mu}$.

By properly plugging an initiator gadget $I_{k}$ (for a well chosen value of $k$ ) and corrector gadgets $C$ onto the generator gadget $G_{m}$, we can, in particular, make sure that the outputs of $G_{m}$ are all labelled 1 by an equitable proper 2-labelling of the whole graph. This is because, by a proper 2labelling assigning label 2 to the outputs, the initiator gadget $I_{k}$ would introduce a huge imbalance in favour of the number of assigned 2's, that is so huge that it cannot be caught up by the labelling freedom of $G_{m}$ and the copies of the corrector gadget $C$.

Once we know that the input and all outputs of $G_{m}$ must be assigned 1 by an equitable proper 2-labelling, the forcing mechanisms in the whole graph then become much easier to track, and it then becomes easier to design an equivalence with a 1-in-3 truth assignment $\phi$ satisfying $F$.

Precise details. The construction of $G$ is as follows. Let us start from the cubic bipartite graph $G_{F}$ modelling the structure of the 3CNF formula $F$. That is, for every variable $x_{i}$ of $F$ we add a variable vertex $v_{i}$ to the graph, for every clause $C_{j}$ of $F$ we add a clause vertex $c_{j}$ to the graph, and, whenever a variable $x_{i}$ belongs to a clause $C_{j}$ in $F$, we add the formula edge $v_{i} c_{j}$ to the graph.

We also add a generator gadget $G_{\mu}$ with $\mu$ outputs to the graph, where $\mu=10(42 m+30 n)$ (where, here and further, $n$ is the number of variables in $F$ and $m$ is the number of clauses in $F$ ) so that we have sufficiently many outputs on hand to perform what follows. We connect some of the outputs and make them adjacent to the clause and variable vertices as follows (see Figure 5 for an illustration for clause vertices):

- For every clause vertex $c_{j}$ :

- We first add three new vertices $e_{j}, f_{j}, g_{j}$, joined via the edges $e_{j} f_{j}, f_{j} g_{j}$, and $e_{j} g_{j}$ to form a triangle. We now identify $e_{j}$ and the degree- 1 vertex of each of 4 unused outputs of $G_{\mu}$. Similarly, we identify $f_{j}$ and the degree- 1 vertex of each of 4 unused outputs of $G_{\mu}$. We next identify $g_{j}$ and the degree- 1 vertex of each of 2 unused outputs of $G_{\mu}$. We finally add the edge $g_{j} c_{j}$ to the graph.

- We then add three new vertices $e_{j}^{\prime}, f_{j}^{\prime}, g_{j}^{\prime}$, forming a triangle. We then identify $e_{j}^{\prime}$ and 5 unused outputs of $G_{\mu}$ as above, $f_{j}^{\prime}$ and 5 unused outputs of $G_{\mu}$, and $g_{j}^{\prime}$ and 3 unused outputs of $G_{\mu}$. We finally add the edge $g_{j}^{\prime} c_{j}$ to the graph.

- We finally add three new vertices $e_{j}^{\prime \prime}, f_{j}^{\prime \prime}, g_{j}^{\prime \prime}$, forming a triangle. We then identify $e_{j}^{\prime \prime}$ and 7 unused outputs of $G_{\mu}$ as above, $f_{j}^{\prime \prime}$ and 7 unused outputs of $G_{\mu}$, and $g_{j}^{\prime \prime}$ and 5 unused 
outputs of $G_{\mu}$. We finally add the edge $g_{j}^{\prime \prime} c_{j}$ to the graph.

- For every variable vertex $v_{i}$ :

- We first add three new vertices $r_{i}, s_{i}, t_{i}$ joined to form a triangle $\left(r_{i}, s_{i}, t_{i}, r_{i}\right)$. We then identify $r_{i}$ and 5 unused outputs of $G_{\mu}$ as above. Similarly, we then identify $s_{i}$ and 5 unused outputs of $G_{\mu}$. Then, we identify $t_{i}$ and 3 unused outputs of $G_{\mu}$. Finally we add the edge $t_{i} v_{i}$ to the graph.

- We then add three new vertices $r_{i}^{\prime}, s_{i}^{\prime}, t_{i}^{\prime}$ joined to form a triangle $\left(r_{i}^{\prime}, s_{i}^{\prime}, t_{i}^{\prime}, r_{i}^{\prime}\right)$. We then identify $r_{i}^{\prime}$ and 6 unused outputs of $G_{\mu}$ as above. Similarly, we then identify $s_{i}^{\prime}$ and 6 unused outputs of $G_{\mu}$. Then, we identify $t_{i}^{\prime}$ and 4 unused outputs of $G_{\mu}$. Finally we add the edge $t_{i}^{\prime} v_{i}$ to the graph.

- Finally, we identify $v_{i}$ with the degree-1 vertex of one unused output of $G_{\mu}$.

Note that there are, at this point, a total of $\beta=3 m+12 m+8 n=15 m+8 n$ edges in the graph that are not part of $G_{\mu}$. More precisely, $3 \mathrm{~m}$ of these edges are edges of $G_{F}$, i.e., formula edges, $12 \mathrm{~m}$ of these edges are part or incident to the triangles added above and joined to the clause vertices, while $8 n$ of these edges are part or incident to the triangles joined to the variable vertices. We refer to this graph, that is, the current one with $\beta$ edges, and that contains none of the edges of $G_{\mu}$, as $G_{F}^{\prime}$.

Note that since $\mu=10(42 m+30 n)$, then, at this point, only $\frac{1}{10} \mu$ outputs of $G_{\mu}$ have been used. To each of the $\frac{9}{10} \mu$ unused outputs of $G_{\mu}$, we plug a new copy of the corrector gadget $C$. Now, we add to the graph an initiator gadget $I_{\alpha}$ of length $\alpha$ that we plug to the input of $G_{\mu}$, where $\alpha$ is chosen to be the unique integer such that

$$
2 \leq((6 \alpha+1)+(32 \mu-31))-(4 \alpha+(56 \mu-56)+\beta) \leq 3 .
$$

The whole resulting graph is our $G$, whose input is the input of $I_{\alpha}$. Clearly, $G$ is obtained in polynomial time from $F$.

Claim 2.9. Let $\ell$ be a proper 2-labelling of $G$. If the input is assigned label 2 , then $\ell$ cannot be equitable.

Proof of the claim. Assume this is wrong, and consider $\ell$ an equitable proper 2-labelling of $G$ assigning label 2 to the input. We investigate how many 1's and 2's must be assigned to several of the different gadgets that were plugged together to build $G$.

- Regarding the initiator gadget $I_{\alpha}$ of length $\alpha$ used in the construction of $G$, by Theorem 2.3 we get that $\ell$ must assign label 1 to exactly $4 \alpha$ edges and label 2 to exactly $6 \alpha+1$ edges.

- By Theorem 2.7, in $G$ the input of each of the $\frac{9}{10} \mu$ copies of the corrector gadget $C$ have their input labelled 2 by $\ell$, since it coincides with an output of $G_{\mu}$ whose input is labelled 2 (by Theorem 2.3, since the output of $I_{\alpha}$ and the input of $G_{\mu}$ coincide). By Theorem 2.4, in each of these copies of $C$, there are 2 edges labelled 1 and 7 edges labelled 2 .

Omitting all of the contributions of the corrector gadgets, we can state that there are, at this point, at least $2 \alpha+1$ more 2's than 1's. This imbalance must be fixed via the labelling of the other edges of $G_{\mu}$ (i.e., not the input of $G_{\mu}$ ) and of $G_{F}^{\prime}$. By Theorem 2.7, at most $56 \mu-56$ edges of $G_{\mu}$ can be assigned label 1 (which yield at least $32 \mu-31$ edges of $G_{\mu}$ labelled 2, due to the number of edges of $G_{\mu}$ ), while the number of edges of $G_{F}^{\prime}$ is $\beta$. By our choice of $\alpha$, it is then impossible that the number of assigned 1's by $\ell$ catches up with the number of assigned 2's. This contradicts the equitability of $\ell$.

Towards establishing the equivalence with a 1-in-3 truth assignment $\phi$ satisfying $F$, let us now see how a proper 2-labelling $\ell$ of $G$ assigning label 1 to the input behaves. We start off by pointing out the following property of the triangles we have joined to the clause and variable vertices. 
Claim 2.10. Let $\gamma \geq 4$. Let $H$ be any graph with a triangle $(u, v, w, u)$ and an edge $x w$ (where $x \notin\{u, v\}$ ), and $\ell$ be a partial proper 2-labelling of $H$. Assume only the edges $u v, v w, w u$, and $x w$ remain to be labelled, that the partial colour of $u$ and $v$ is $\gamma-2$, and that the partial colour of $w$ is $\gamma-4$. Then, in every proper extension of $\ell$ to $u v, v w, w u$, and $x w$, we have $\ell(u v)=2, \ell(x w)=1$, and $c_{\ell}(w)=\gamma$.

Proof of the claim. Since only $u v, v w, w u$, and $x w$ remain to be labelled, and $u$ and $v$ currently have the same partial colour, so that $c_{\ell}(u) \neq c_{\ell}(v)$ we have, say, $\ell(u w)=1$ and $\ell(v w)=2$. If $\ell(u v)=1$, then we get $c_{\ell}(u)=\gamma, c_{\ell}(v)=\gamma+1$, while the partial colour of $w$ is currently $\gamma-1$. It is then impossible to assign a correct label to $x w$, i.e., so that $c_{\ell}(w) \notin\{\gamma, \gamma+1\}=\left\{c_{\ell}(u), c_{\ell}(v)\right\}$. So, we have $\ell(u v)=2$, in which case $c_{\ell}(u)=\gamma+1$ and $c_{\ell}(v)=\gamma+2$. As above, the partial colour of $w$ is currently $\gamma-1$, and, so that $c_{\ell}(w) \notin\{\gamma+1, \gamma+2\}$, we must set $\ell(x w)=1$. Then, $c_{\ell}(w)=\gamma$.

Claim 2.10, applied to the structure of $G$ (and more precisely to that of $G_{F}^{\prime}$ ), yields the following.

Claim 2.11. For any proper 2-labelling $\ell$ of $G$ assigning label 1 to the input:

- For each clause vertex $c_{j}$, exactly one of its three incident formula edges is assigned label 1. Hence, $c_{\ell}\left(c_{j}\right)=8$.

- For each variable vertex $v_{i}$, either all three of its incident formula edges are assigned label 1 , or they are all assigned label 2. Hence, $c_{\ell}\left(v_{i}\right) \in\{6,9\}$.

- The number of edges in $G_{F}^{\prime}$ that are assigned label 1 by $\ell$ is $7 m+4 n$, while the number of edges assigned label 2 is $8 m+4 n$.

Proof of the claim. Let $\ell$ be such a labelling of $G$. By Theorems 2.3 and 2.7, all outputs of $G_{m}$ must also be labelled 1 by $\ell$.

- Consider any clause vertex $c_{j}$ of $G$, and, in particular, the neighbouring triangle $\left(e_{j}, f_{j}, g_{j}\right)$. Note that all the conditions are met to apply Claim 2.10. Similarly, this claim applies to the two triangles $\left(e_{j}^{\prime}, f_{j}^{\prime}, g_{j}^{\prime}\right)$ and $\left(e_{j}^{\prime \prime}, f_{j}^{\prime \prime}, g_{j}^{\prime \prime}\right)$. From the claim, we get that $\ell\left(g_{j} c_{j}\right)=\ell\left(g_{j}^{\prime} c_{j}\right)=$ $\ell\left(g_{j}^{\prime \prime} c_{j}\right)=1, c_{\ell}\left(g_{j}\right)=6, c_{\ell}\left(g_{j}^{\prime}\right)=7$, and $c_{\ell}\left(g_{j}^{\prime \prime}\right)=9$. Since $c_{j}$ is incident to only three other edges, formula ones, one of them must be labelled 1 while the other two must be labelled 2 so that $c_{\ell}\left(c_{j}\right) \notin\{6,7,9\}$. Then, $c_{\ell}\left(c_{j}\right)=8$.

- Consider any variable vertex $v_{i}$ of $G$. By the same arguments, we have $\ell\left(t_{i} v_{i}\right)=\ell\left(t_{i}^{\prime} v_{i}\right)=1$, $c_{\ell}\left(t_{i}\right)=7$, and $c_{\ell}\left(t_{i}^{\prime}\right)=8$. Consequently, the three remaining (formula) edges incident to $v_{i}$ must either all be labelled 1 by $\ell$, so that $c_{\ell}\left(v_{i}\right)=6$, or all be labelled 2 , so that $c_{\ell}\left(v_{i}\right)=9$ (recall that $v_{i}$ is also incident to an output of $G_{\mu}$ labelled 1 ). These are the only two possibilities so that $c_{\ell}\left(v_{i}\right) \notin\{7,8\}$.

The last part of the statement follows from Claim 2.10 and the arguments above. This concludes the proof. Note, in particular, that a consequence is that we have $c_{\ell}\left(c_{j}\right) \neq c_{\ell}\left(v_{i}\right)$ for every clause vertex $c_{j}$ and variable vertex $v_{i}$.

Claim 2.11 gives us a direct equivalence between finding a proper 2-labelling of $G$ where the input is labelled 1 and a 1-in-3 truth assignment to the variables of $F$. Indeed, consider a proper 2-labelling $\ell$ of $G$ assigning label 1 to the input. We regard the fact that $\ell\left(v_{i} c_{j}\right)=1$ (respectively $\ell\left(v_{i} c_{j}\right)=2$ ) as having, in $F$, variable $v_{i}$ bringing truth value true (respectively false) to clause $C_{j}$. The condition in the first item of Claim 2.11 depicts the fact that, by a 1-in-3 truth assignment of $F$, a clause is considered satisfied only if it has exactly one true variable. The condition in the second item depicts the fact that, by a truth assignment, a variable brings the same truth value to all of the clauses that contain it. Thus, we can design a 1-in-3 truth assignment $\phi$ to the variables of $F$ from $\ell$, and vice versa. 
Thus, $F$ is 1-in-3 satisfiable if and only if $G$ admits proper 2-labellings where the input is labelled 1. By Claim 2.9, all equitable proper 2-labellings of $G$ (if any) must assign label 1 to the input. Thus, to prove that $F$ is 1-in-3 satisfiable if and only if $G$ admits equitable proper 2-labellings, it remains to show that $G$ admits proper 2-labellings assigning label 1 to the input if and only if it admits equitable ones assigning label 1 to the input. Since every equitable proper labelling is a proper labelling, all that remains is to prove the following claim.

Claim 2.12. If $G$ admits proper 2-labellings where the input is assigned label 1 , then $G$ admits equitable proper 2-labellings where the input is assigned label 1.

Proof of the claim. Let us consider a proper 2-labelling $\ell$ of $G$ assigning label 1 to the input, obtained as follows. From Theorems 2.3 and 2.7, we know that all outputs of $G_{\mu}$ must also be assigned label 1. We propagate $\ell$ in $I_{\alpha}, G_{\mu}$, and $G_{F}^{\prime}$ while guaranteeing the following properties:

- In $I_{\alpha}$, exactly $4 \alpha$ edges are assigned label 2 by $\ell$, while $6 \alpha+1$ edges are assigned label 1 . This is actually the only way to propagate $\ell$ in $I_{\alpha}$, recall Theorem 2.3. Thus, here, there are $2 \alpha+1$ more assigned 1's than assigned 2's.

- In $G_{\mu}$, the number of assigned 1's is as small as possible, i.e., is $32 \mu-31$. In that case, the number of assigned 2's is $56 \mu-56$. Such a labelling can be achieved by Theorem 2.7.

- In $G_{F}^{\prime}$, the number of assigned 2's is $m$ more than the number of assigned 1's. By Claim 2.11 and since $G$ admits a proper 2-labelling where the input is assigned label 1, this property is attainable (while maintaining that the labelling is proper) and actually has to hold.

To summarise the above, at this point, the number of assigned 1's is $((6 \alpha+1)+(32 \mu-$ $31))-(4 \alpha+(56 \mu-56)+m)$ more than the number of assigned 2's. Recall that $\alpha$ was chosen as the unique integer such that $2 \leq((6 \alpha+1)+(32 \mu-31))-(4 \alpha+(56 \mu-56)+\beta) \leq 3$. Thus, $\beta-m+2 \leq((6 \alpha+1)+(32 \mu-31))-(4 \alpha+(56 \mu-56)+m) \leq \beta-m+3$, and hence, the number of assigned 1's we have considered is either $14 m+8 n+2$ or $14 m+8 n+3$ more than the number of assigned 2's (recall that $\beta=15 m+8 n$ ). It remains to consider the $\frac{9}{10} \mu=9(42 m+30 n)$ copies of the corrector gadget $C$ in $G$. This means that the number of copies of $C$ in $G$ is much bigger than $14 m+8 n+3$. By Theorem 2.4, we can propagate $\ell$ to some copies of $C$ so that 6 edges are assigned label 2 and 3 edges are assigned label 1. This way, the number of assigned 2's we have considered thus far catches up with the number of assigned 1's.

For the remaining copies of $C$, we can assume that $\ell$ roughly alternates propagating following the two labelling possibilities described in Theorem 2.4 when the input is labelled 1, so that the number of assigned 2's we have considered remains close yet slightly bigger than the number of assigned 1's. If, eventually, $\ell$ is not equitable because the number of assigned 2's is slightly bigger than the number of assigned 1's, then we can freely switch from 2 to 1 the labels assigned to some edges of, e.g., triangles in the copies of $T_{2}$ in some of the spreading gadgets $G^{\curlywedge}$ in $G_{\mu}$. Recall that all these edges are indeed currently assigned label 2 (since we have minimised the number of assigned 1's in $G_{\mu}$ ).

Something to take into consideration is that the labelling of $G_{\mu}$ we have considered above, i.e., the one minimising the number of 1's, does not comply with the two labelling schemes of the corrector gadget $C$. Indeed, when the spreading gadget $G^{\curlywedge}$ is labelled so that the input is labelled 1 and the number of 1 's is minimised, note that the vertices $u_{9}$ and $u_{12}$ must have colour 3 , which is not compatible with the labelling of $C$ in Figure 2(b). In this case, it is necessary to make $u_{9}$ (or $u_{12}$ ) have colour 2 (so that they comply with the desired labelling of $C$ ) by just changing to 1 the label of an incident edge labelled 2. This consequently makes the number of 2 's increase, which must be taken into consideration for deciding how to label the next copies of $C$.

Eventually, $\ell$ is equitable.

To finish off the proof, we prove that, regardless of whether $F$ is 1-in-3 satisfiable, there always exist proper 2-labellings of $G$. In other words, we always have $\chi_{\Sigma}(G)=2$. 
Claim 2.13. There exist proper 2-labellings of $G$.

Proof of the claim. We show that $G$ admits proper 2-labellings $\ell$ where the input is labelled 2 . Recall that we do not care about equitability here. By Theorem 2.3, the output of $I_{\alpha}$, which is the input of $G_{\mu}$, must be labelled 2 when its input is labelled 2. In turn, by Theorem 2.7, the outputs of $G_{\mu}$ must be labelled 2 as well. Some of these outputs are the input of corrector gadgets. From Theorems 2.3, 2.4, and 2.7, we get that there do exist partial proper 2-labellings $\ell$ of these gadgets in $G$ such that no conflicts arise.

It remains to prove that such a partial labelling $\ell$ can properly be extended to the edges of $G_{F}^{\prime}$. We demonstrate the arguments for one triangle $\left(e_{j}, f_{j}, g_{j}, e_{j}\right)$ adjacent to a clause vertex $c_{j}$, but the arguments are identical regarding the other triangles and the variable vertices. Because $e_{j}$ and $f_{j}$ are incident to 4 edges of $G_{\mu}$, which are all labelled 2 by the arguments above, $e_{j}$ and $f_{j}$ already have partial colour 8. Let us assign label 2 to $e_{j} f_{j}$ and $f_{j} g_{j}$, and label 1 to $e_{j} g_{j}$. This gives $c_{\ell}\left(e_{j}\right)=11 \neq c_{\ell}\left(f_{j}\right)=12$. At this point, $g_{j}$ has partial colour 7 (3 from the labelling of $e_{j} g_{j}$ and $f_{j} g_{j}$, and 4 from the two outputs of $\left.G_{\mu}\right)$. Let us assign label 1 to $g_{j} c_{j}$, so that $c_{\ell}\left(g_{j}\right)=8$. Note that no two of $e_{j}, f_{j}$, and $g_{j}$ are in conflict, and also their colour is so big that no conflict with adjacent vertices in $G_{\mu}$ can arise.

By repeating these arguments, for every clause vertex $c_{j}$, its incident edges $c_{j} g_{j}, c_{j} g_{j}^{\prime}$, and $c_{j} g_{j}^{\prime \prime}$ are labelled 1 , while $g_{j}, g_{j}^{\prime}$, and $g_{j}^{\prime \prime}$ have colour at least 8 . In particular, $c_{j}$ has partial colour 3 at this point. Similarly, for every variable vertex $v_{i}$ its incident edges $v_{i} t_{i}$ and $v_{i} t_{i}^{\prime}$ are labelled 1 , and $t_{i}$ and $t_{i}^{\prime}$ have colour at least 10. Recall that an output of $G_{\mu}$, which is labelled 2, is also attached to $v_{i}$. Then, the partial colour of $v_{i}$ is 4 at this point. Let us finish the construction of the labelling $\ell$ by assigning label 1 to every formula edge. Since every clause vertex $c_{j}$ and variable vertex $v_{i}$ is incident to exactly three such edges, we get that $c_{\ell}\left(c_{j}\right)=6$ and $c_{\ell}\left(v_{i}\right)=7$ for every clause vertex $c_{j}$ and every variable vertex $v_{i}$. Then, neither clause vertices nor variable vertices get in conflict with any of their neighbours. In particular, no clause vertex gets in conflict with a variable vertex. Then, $\ell$ is proper, as desired.

This concludes the proof.

Another interpretation of Theorem 2.8 is that, independently of whether Conjecture 1.2 is true or not, determining $\overline{\chi_{\Sigma}}(G)$ is an NP-hard problem for a given graph $G$. It is also worth mentioning that, in our reduction, the reduced graphs $G$ we construct should always verify $\overline{\chi_{\Sigma}}(G) \leq 3$. This can be seen by noting that all gadgets and structures we have added to $G$ themselves admit many equitable proper 3-labellings, some of which could possibly be combined to yield one of $G$.

\section{$3 \quad$ Bipartite graphs $G$ with $\chi_{\Sigma}(G)<\overline{\chi_{\Sigma}}(G)$}

In this section, we investigate the existence of bipartite graphs $G$ with $\chi_{\Sigma}(G)<\overline{\chi_{\Sigma}}(G)$. In Section 3.1, we first focus on bipartite graphs $G$ with $\chi_{\Sigma}(G)=3$, as they stand as good candidates of graphs that could have $\overline{\chi_{\Sigma}}(G)>3$. We prove that, actually, $\overline{\chi_{\Sigma}}(G)=3$ holds for all these graphs. In Section 3.2, we then study the existence of bipartite graphs $G$ with $\chi_{\Sigma}(G)=2$ and $\overline{\chi_{\Sigma}}(G)=3$. We provide operations for building infinitely many such graphs.

\subsection{Bipartite graphs $G$ with $\chi_{\Sigma}(G)=3$}

A precise characterisation of bipartite graphs $G$ with $\chi_{\Sigma}(G)=3$ was provided by Thomassen, Wu, and Zhang in [12], where they proved that these graphs, called odd multi-cacti, can be obtained as follows. The definition is recursive.

- Any cycle $C_{4 k+2}$ with length $4 k+2(k \geq 1)$ with its edges being properly coloured with red and green (i.e., no two subsequent edges have the same colour) is an odd multi-cactus. 
- Given an odd multi-cactus $G$ with edges coloured red and green, another odd multi-cactus is obtained when considering any green edge $u v$ of $G$, and joining $u v$ by a new path $P$ of length $4 k+1(k \geq 1)$, where the edges of $P$ are properly coloured with red and green in such a way that the two end-edges (i.e., incident to $u$ and $v$ ) are coloured red.

In the next result, we prove that for every odd multi-cactus $G$, we have $\chi_{\Sigma}(G)=\overline{\chi_{\Sigma}}(G)=3$.

Theorem 3.1. If $G$ is an odd multi-cactus, then $\overline{\chi \Sigma}(G)=3$.

Proof. The proof is by induction on the number of vertices of $G$. The base case corresponds to $G$ being $C_{6}$, the cycle of length 6 , which is the smallest odd multi-cactus. We first prove a more general case, namely that the claim is true whenever $G$ is a cycle with length at least 6 congruent to 2 modulo 4 .

Let $G$ be a cycle with length at least 6 congruent to 2 modulo 4 . In this case, an equitable proper 3-labelling can be obtained as follows. Traverse the successive edges of $G$ starting from an arbitrary one, and assign labels $1,2,3,1,2,3, \ldots$ going along until all edges are labelled. Note that, doing so, at any moment of the procedure the resulting partial labelling is equitable.

- If the length of $G$ is congruent to 0 or 1 modulo 3, then, by Observation 2.1, the resulting labelling is proper. This is because no two edges at distance 2 receive the same label, which is the only colour conflict that can occur in a path.

- If the length of $G$ is congruent to 2 modulo 3 (the smallest such graph is $C_{14}$ ), then we get a conflict because of the last two edges that were labelled $l_{1}=1$ and $l_{2}=2$ respectively, which are each at distance 2 from an edge with the same label. In this situation, we change $l_{1}$ into 3 and $l_{2}$ into 1 . Note that no conflict remains now. Furthermore, the labelling remains equitable (the number of assigned 2's is one less than the numbers of assigned 1's and 3's, which are equal).

For the general case: suppose that all odd multi-cacti with order at most some $x-1$ admit an equitable proper 3-labelling, and let us consider odd multi-cacti with order $x$. If $x \not \equiv 2 \bmod 4$, then, by construction, there exist no such graphs on $x$ vertices, and the claim is true. Thus, we assume that $x \equiv 2 \bmod 4$.

Let $G$ be an odd multi-cactus with $x$ vertices. Since $G$ can be assumed to be different from a cycle, it was obtained from a cycle of length 2 modulo 4 by repeated path attachments onto green edges. Due to the structure of $G$, it can be noted that there has to exist a green edge $u v$ where:

1. there exist $p \geq 1$ disjoint paths $P_{1}, \ldots, P_{p}$ joining $u$ and $v$, all of whose inner vertices have degree 2 , and

2. the graph $G^{\prime}$ obtained by removing the inner vertices of the paths $P_{1}, \ldots, P_{p}$ from $G$ is an odd multi-cactus where $u$ and $v$ have degree 2 .

First off, it can be assumed that all of the $P_{i}$ 's have length 5 . This is a consequence of the following more general result:

Claim 3.2. Let $P_{9}=\left(v_{1}, \ldots, v_{9}\right)$ be the path of length 8 , and assume we are given a partial proper 3-labelling $\ell^{\prime}$ of $P_{9}$ where only the four edges $v_{1} v_{2}, v_{2} v_{3}, v_{7} v_{8}$, and $v_{8} v_{9}$ are labelled, so that $\ell^{\prime}\left(v_{1} v_{2}\right) \neq \ell^{\prime}\left(v_{7} v_{8}\right)$ and $\ell^{\prime}\left(v_{2} v_{3}\right) \neq \ell^{\prime}\left(v_{8} v_{9}\right)$. Then, for any permutation $\{\alpha, \beta, \gamma\}$ of $\{1,2,3\}$, it is possible to extend $\ell^{\prime}$ to a proper 3 -labelling $\ell$ of $P_{9}$ where two of $v_{3} v_{4}, v_{4} v_{5}, v_{5} v_{6}$, and $v_{6} v_{7}$ are labelled $\alpha$, one of these edges is labelled $\beta$, and one of these edges is labelled $\gamma$.

Proof of the claim. So that a labelling of $P_{9}$ is proper, we must only ensure that every two edges at distance 2 receive distinct labels, recall Observation 2.1. In particular, this implies that labelling $v_{3} v_{4}$ and $v_{5} v_{6}$ can be done independently from labelling $v_{4} v_{5}$ and $v_{6} v_{7}$. Then, we will be done if 
we can prove that labels $\alpha$ and $\beta$ can correctly be assigned to $v_{3} v_{4}$ and $v_{5} v_{6}$, while labels $\alpha$ and $\gamma$ can correctly be assigned to $v_{4} v_{5}$ and $v_{6} v_{7}$.

Without loss of generality, let us assume we want to assign labels $\alpha$ and $\beta$ to $v_{3} v_{4}$ and $v_{5} v_{6}$. Note that, at this point, $\ell\left(v_{3} v_{4}\right)$ must only differ from $\ell\left(v_{1} v_{2}\right)$. Let us assume that we can assign $\ell\left(v_{3} v_{4}\right)=\alpha$ without there being a conflict with $\ell\left(v_{1} v_{2}\right)$, i.e., $\ell\left(v_{1} v_{2}\right) \neq \alpha$. If no conflict arises upon setting $\ell\left(v_{5} v_{6}\right)=\beta$, then we are done. Otherwise, it means $\ell\left(v_{7} v_{8}\right)=\beta$. In that situation, let us instead set $\ell\left(v_{5} v_{6}\right)=\alpha$ and $\ell\left(v_{3} v_{4}\right)=\beta$. If this raises a conflict, this must be because $\ell\left(v_{1} v_{2}\right)=\beta$. But then, we deduce that $\ell\left(v_{1} v_{2}\right)=\ell\left(v_{7} v_{8}\right)=\beta$, a contradiction.

Indeed, assume, without loss of generality, that $P_{1}$ has length $4 k+1$ for some $k \geq 2$. Let us denote by $\left(u, v_{1}, \ldots, v_{4 k}, v\right)$ the successive vertices of $P_{1}$ from $u$ to $v$. Let $G^{\prime}$ be the graph obtained from $G$ by removing the edges $v_{2} v_{3}, v_{3} v_{4}, v_{4} v_{5}, v_{5} v_{6}, v_{6} v_{7}$ and joining the vertices $v_{2}$ and $v_{7}$ by an edge $e$. Note that $G^{\prime}$ is an odd multi-cactus since we have essentially contracted a path of length $4 k+1$ (with $k \geq 2$ ) into a path of length $4(k-1)+1$. Then, by the induction hypothesis, there is an equitable proper 3-labelling $\ell^{\prime}$ of $G^{\prime}$. By definition, note that $\ell^{\prime}\left(u v_{1}\right) \neq \ell^{\prime}(e), \ell^{\prime}(e) \neq \ell^{\prime}\left(v_{8} v_{9}\right)$ (or $\ell^{\prime}(e) \neq \ell^{\prime}\left(v_{8} v\right)$ if $\left.k=2\right)$, and $\ell^{\prime}\left(v_{1} v_{2}\right) \neq \ell^{\prime}\left(v_{7} v_{8}\right)$. To extend $\ell^{\prime}$ to an equitable proper 3-labelling $\ell$ of $G$, for every edge that is both in $G$ and $G^{\prime}$ we first infer the label by $\ell^{\prime}$ to $\ell$. We then set $\ell\left(v_{2} v_{3}\right)$ to $\ell^{\prime}(e)$. Note that no conflict arises in $G$ by this partial labelling. Furthermore, since $\ell^{\prime}$ is equitable, so is $\ell$. Now, consider $\{\alpha, \beta, \gamma\}$ a permutation of $\{1,2,3\}$ such that every two of $\mathrm{nb}_{\ell^{\prime}}(\alpha)+2, \operatorname{nb}_{\ell^{\prime}}(\beta)+1$, and $\operatorname{nb}_{\ell^{\prime}}(\gamma)+1$ differ by at most 1 . Such an $\{\alpha, \beta, \gamma\}$ exists since $\ell^{\prime}$ is equitable. By Claim 3.2, the current $\ell$ can be extended to the edges $v_{3} v_{4}, v_{4} v_{5}, v_{5} v_{6}, v_{6} v_{7}$ so that a proper 3-labelling of $G$ results, and that this can be done by assigning label $\alpha$ twice, and each of $\beta$ and $\gamma$ once. By our choice of $\alpha, \beta, \gamma$, such a resulting labelling is also equitable.

Then, we can assume all $P_{i}$ 's have length exactly 5 . For every $i \in\{1, \ldots, p\}$, let us set $P_{i}=\left(u, v_{1}^{i}, \ldots, v_{4}^{i}, v\right)$. Let us also denote by $u^{\prime}$ the second neighbour (different from $v$ ) of $u$ in $G^{\prime}$, and by $v^{\prime}$ the second neighbour (different from $u$ ) of $v$ in $G^{\prime}$. Our goal is to extend $\ell^{\prime}$ to the edges of the $P_{i}$ 's so that no conflict arises, and the resulting 3-labelling $\ell$ of $G$ is proper. To begin, consider $\{\alpha, \beta, \gamma\}$ a permutation of $\{1,2,3\}$. The choice of $\alpha, \beta$, and $\gamma$ can be done in such a way that the ensuing labelling $\ell$ is equitable. Precisely, if $\mathrm{nb}_{\ell^{\prime}}(1)=\mathrm{nb}_{\ell^{\prime}}(2)=\mathrm{nb}_{\ell^{\prime}}(3)$ or $\mathrm{nb}_{\ell^{\prime}}(1)+1=\mathrm{nb}_{\ell^{\prime}}(2)=\mathrm{nb}_{\ell^{\prime}}(3)$ or $\mathrm{nb}_{\ell^{\prime}}(1)=\mathrm{nb}_{\ell^{\prime}}(2)=\mathrm{nb}_{\ell^{\prime}}(3)-1$, then $(\alpha, \beta, \gamma)=(1,2,3)$, else if $\mathrm{nb}_{\ell^{\prime}}(1)-1=\mathrm{nb}_{\ell^{\prime}}(2)=\mathrm{nb}_{\ell^{\prime}}(3)$ or $\mathrm{nb}_{\ell^{\prime}}(1)=\mathrm{nb}_{\ell^{\prime}}(2)+1=\mathrm{nb}_{\ell^{\prime}}(3)$, then $(\alpha, \beta, \gamma)=(2,3,1)$, and if $\mathrm{nb}_{\ell^{\prime}}(1)=\mathrm{nb}_{\ell^{\prime}}(2)=\mathrm{nb}_{\ell^{\prime}}(3)+1 \operatorname{or}_{\mathrm{nb}_{\ell^{\prime}}}(1)=\mathrm{nb}_{\ell^{\prime}}(2)-1=\mathrm{nb}_{\ell^{\prime}}(3)$, then $(\alpha, \beta, \gamma)=(3,1,2)$. For all $1 \leq i \leq p$ and for any $a, b \in P_{i}$ such that $a b \in E(G)$, it is easy to verify that $c_{\ell}(a) \neq c_{\ell}(b)$, for any of the labellings $\ell$ proposed below.

In what follows, let $x$ be any vertex in $X=N(u) \bigcap_{i=1}^{p} P_{i}$, let $y$ be any vertex in $Y=N(v) \bigcap_{i=1}^{p} P_{i}$, and let $w$ be any vertex in $W=(N(u) \cup N(v)) \bigcap_{i=1}^{p} P_{i}$. Also, for all $1 \leq i \leq p$, let $\ell\left(P_{i}\right)=$ $\left(\ell\left(u v_{1}^{i}\right), \ell\left(v_{1}^{i} v_{2}^{i}\right), \ell\left(v_{2}^{i} v_{3}^{i}\right), \ell\left(v_{3}^{i} v_{4}^{i}\right), \ell\left(v_{4}^{i} v\right)\right)$.

Case $p=2$ :

All the possible subcases are illustrated in Table 1. Note that, in all of these subcases, the labelling $\ell$ has the property that $\sum_{x \in X} \ell(u x)=\sum_{y \in Y} \ell(v y)$, and so, $c_{\ell}(u) \neq c_{\ell}(v)$ since $c_{\ell^{\prime}}(u) \neq c_{\ell^{\prime}}(v)$ by the inductive hypothesis. Furthermore, note that the maximum colour of any vertex $w \in W$ is 6 , no matter the labelling. Lastly, it may seem that the subcase $c_{\ell^{\prime}}(u)+\alpha+\beta \neq c_{\ell^{\prime}}\left(u^{\prime}\right)$, $c_{\ell^{\prime}}(v)+\alpha+\beta=c_{\ell^{\prime}}\left(v^{\prime}\right)$ has not been treated, but it is actually symmetric to the subcase where $c_{\ell^{\prime}}(u)+\alpha+\beta=c_{\ell^{\prime}}\left(u^{\prime}\right), c_{\ell^{\prime}}(v)+\alpha+\beta \neq c_{\ell^{\prime}}\left(v^{\prime}\right)$, which has been treated through subcases 2-4 in Table 1.

Case $p \geq 3$ : 


\begin{tabular}{|c|c|c|}
\hline $\begin{array}{l}\text { Subcase } \\
\text { conditions }\end{array}$ & $\begin{array}{l}\text { Labelling of } \\
\text { paths } P_{1} \text { and } P_{2}\end{array}$ & $\begin{array}{c}\text { No conflicts between } u \text { ( } v \text { resp.) } \\
\text { and any of its neighbours in } P_{1} \text { or } P_{2}\end{array}$ \\
\hline $\begin{array}{l}c_{\ell^{\prime}}(u)+\alpha+\beta \neq c_{\ell^{\prime}}\left(u^{\prime}\right), \\
c_{\ell^{\prime}}(v)+\alpha+\beta \neq c_{\ell^{\prime}}\left(v^{\prime}\right)\end{array}$ & $\begin{array}{l}\ell\left(P_{1}\right)=(\alpha, \beta, \gamma, \alpha, \beta) \\
\ell\left(P_{2}\right)=(\beta, \alpha, \gamma, \gamma, \alpha)\end{array}$ & $\begin{aligned} \text { If } \alpha=1: c_{\ell}(w) \leq 4 \& c_{\ell}(u), c_{\ell}(v) \geq 5 \\
\\
\text { If } \alpha=2: c_{\ell}(u), c_{\ell}(v) \geq 7 . \\
\text { If } \alpha=3: c_{\ell}(w) \leq 5 \& c_{\ell}(u), c_{\ell}(v) \geq 6 .\end{aligned}$ \\
\hline $\begin{array}{c}c_{\ell^{\prime}}(u)+\alpha+\beta=c_{\ell^{\prime}}\left(u^{\prime}\right), \\
c_{\ell^{\prime}}(v)+2 \alpha \neq c_{\ell^{\prime}}\left(v^{\prime}\right) \\
c_{\ell^{\prime}}(v)+2 \alpha \neq \alpha+\gamma\end{array}$ & $\begin{array}{l}\ell\left(P_{1}\right)=(\alpha, \beta, \beta, \gamma, \alpha) \\
\ell\left(P_{2}\right)=(\alpha, \beta, \gamma, \gamma, \alpha)\end{array}$ & $\begin{array}{c}\text { If } \alpha=1: c_{\ell}(x)=3 \& c_{\ell}(u) \geq 4 . \\
\text { If } \alpha=2: c_{\ell}(x)=5 \& c_{\ell}(u) \geq 6 . \\
\text { If } \alpha=3: c_{\ell}(u) \geq 8 .\end{array}$ \\
\hline $\begin{array}{c}c_{\ell^{\prime}}(u)+\alpha+\beta=c_{\ell^{\prime}}\left(u^{\prime}\right) \\
c_{\ell^{\prime}}(v)+2 \alpha \neq c_{\ell^{\prime}}\left(v^{\prime}\right) \\
c_{\ell^{\prime}}(v)+2 \alpha=\alpha+\gamma\end{array}$ & $\begin{array}{l}\ell\left(P_{1}\right)=(\alpha, \gamma, \beta, \beta, \alpha) \\
\ell\left(P_{2}\right)=(\alpha, \gamma, \gamma, \beta, \alpha)\end{array}$ & $\begin{array}{l}\text { Since } c_{\ell^{\prime}}(u) \neq c_{\ell^{\prime}}(v) \text {, then } \\
\qquad c_{\ell^{\prime}}(u)+2 \alpha \neq \alpha+\gamma\end{array}$ \\
\hline $\begin{array}{c}c_{\ell^{\prime}}(u)+\alpha+\beta=c_{\ell^{\prime}}\left(u^{\prime}\right) \\
c_{\ell^{\prime}}(v)+2 \alpha=c_{\ell^{\prime}}\left(v^{\prime}\right)\end{array}$ & $\begin{array}{l}\ell\left(P_{1}\right)=(\gamma, \alpha, \beta, \gamma, \alpha) \\
\ell\left(P_{2}\right)=(\alpha, \gamma, \beta, \beta, \gamma)\end{array}$ & $\begin{array}{c}\text { If } \alpha=1: c_{\ell}(w) \leq 5 \& c_{\ell}(u), c_{\ell}(v) \geq 6 . \\
\text { If } \alpha=2: c_{\ell}(w) \leq 4 \& c_{\ell}(u), c_{\ell}(v) \geq 5 . \\
\text { If } \alpha=3: c_{\ell}(u), c_{\ell}(v) \geq 7 .\end{array}$ \\
\hline
\end{tabular}

Table 1: The four subcases for $p=2$.

Give the same labellings as in the case $p=2$ for $P_{1}$ and $P_{2}$. For the remainder of the paths, simply label them so that the labelling $\ell$ is equitable (and proper) and so that, for all $3 \leq j \leq p$, we have $\ell\left(u v_{1}^{j}\right)=\ell\left(v v_{4}^{j}\right)$. Note that, in this case, for all $x \in X$, it is not possible for $c_{\ell}(u)=c_{\ell}(x)$. Indeed, if $c_{\ell}(u) \geq 7$, then we are done since, for all $w \in W$, we have that $c_{\ell}(w) \leq 6$. Otherwise, if $c_{\ell}(u)=6$, then, for all $x \in X$, we have that $\ell(u x) \leq 2$, and so, $c_{\ell}(x) \leq 5$. Lastly, if $c_{\ell}(u)=5$ (note that this is the last case since $c_{\ell}(u) \geq 5$ ), then, for all $x \in X$, we have that $\ell(u x)=1$, and so, $c_{\ell}(x) \leq 4$. The same can be said for all $y \in Y$ and $v$.

Case $p=1$ :

All the possible subcases are illustrated in Table 2. Note that, in the first six of these subcases, the labelling $\ell$ has the property that $\ell\left(u v_{1}\right)=\ell\left(v v_{4}\right)$, and so, $c_{\ell}(u) \neq c_{\ell}(v)$ since $c_{\ell^{\prime}}(u) \neq c_{\ell^{\prime}}(v)$ by the inductive hypothesis. Furthermore, note that the logical subcase that would follow the last subcase in Table 2 would be that $c_{\ell^{\prime}}(u)+\alpha=c_{\ell^{\prime}}\left(u^{\prime}\right), c_{\ell^{\prime}}(v)+\beta=c_{\ell^{\prime}}\left(v^{\prime}\right), c_{\ell^{\prime}}(u)+\beta \neq c_{\ell^{\prime}}(v)+\alpha$, $c_{\ell^{\prime}}(v)+\alpha=2 \alpha$, and $c_{\ell^{\prime}}(u)+\beta=\beta+\alpha$. However, this subcase cannot exist since, if $c_{\ell^{\prime}}(v)+\alpha=2 \alpha$, then $c_{\ell^{\prime}}(v)=\alpha$, and if $c_{\ell^{\prime}}(u)+\beta=\beta+\alpha$, then $c_{\ell^{\prime}}(u)=\alpha$, and hence, we have that $c_{\ell^{\prime}}(u)=c_{\ell^{\prime}}(v)$, a contradiction. Lastly, it may seem that the subcase $c_{\ell^{\prime}}(u)+\alpha \neq c_{\ell^{\prime}}\left(u^{\prime}\right), c_{\ell^{\prime}}(v)+\alpha=c_{\ell^{\prime}}\left(v^{\prime}\right)$ has not been treated, but again, it is actually symmetric to the subcase where $c_{\ell^{\prime}}(u)+\alpha=c_{\ell^{\prime}}\left(u^{\prime}\right)$, $c_{\ell^{\prime}}(v)+\alpha \neq c_{\ell^{\prime}}\left(v^{\prime}\right)$, which has been treated through subcases 4-12 in Table 2. This concludes the proof as all of the possible cases have now been covered.

\subsection{Bipartite graphs $G$ with $\chi_{\Sigma}(G)=2$}

We start by introducing two operations, Operations 1 and 2, which, when applied to graphs $G$ with $\overline{\chi_{\Sigma}}(G) \geq 3$, provide more graphs $G^{\prime}$ with $\overline{\chi_{\Sigma}}\left(G^{\prime}\right) \geq 3$.

Theorem 3.3 (Operation 1). Let $G$ be a multigraph with $\overline{\chi_{\Sigma}}(G) \geq 3$. If $G$ has an edge uv with multiplicity at least 2 , then the graph $G^{\prime}$ obtained from $G$ by subdividing one of these edges uv four times verifies $\overline{\chi_{\Sigma}}\left(G^{\prime}\right) \geq 3$.

Proof. Let $G^{\prime}$ be obtained from $G$ by replacing one edge $u v$ with a path $(u, w, x, y, z, v)$ of length 5 . Assume there exists $\ell^{\prime}$, an equitable proper 2-labelling of $G^{\prime}$. Assume that $\ell^{\prime}(u w)=\alpha$ for some 


\begin{tabular}{|c|c|c|}
\hline $\begin{array}{c}\text { Subcase } \\
\text { conditions }\end{array}$ & $\begin{array}{l}\text { Labelling } \\
\text { of path } P_{1}\end{array}$ & $\begin{array}{l}\text { No conflicts between } u \text { ( } v \text { resp.) } \\
\text { and any of its neighbours in } P_{1}\end{array}$ \\
\hline $\begin{array}{l}c_{\ell^{\prime}}(u)+\alpha \neq c_{\ell^{\prime}}\left(u^{\prime}\right), \\
c_{\ell^{\prime}}(v)+\alpha \neq c_{\ell^{\prime}}\left(v^{\prime}\right), \\
c_{\ell^{\prime}}(v)+\alpha \neq \beta+\alpha, \\
c_{\ell^{\prime}}(u)+\alpha \neq \gamma+\alpha\end{array}$ & $\ell\left(P_{1}\right)=(\alpha, \gamma, \beta, \beta, \alpha)$ & By the conditions of the subcase. \\
\hline $\begin{array}{l}c_{\ell^{\prime}}(u)+\alpha \neq c_{\ell^{\prime}}\left(u^{\prime}\right), \\
c_{\ell^{\prime}}(v)+\alpha \neq c_{\ell^{\prime}}\left(v^{\prime}\right), \\
c_{\ell^{\prime}}(v)+\alpha \neq \beta+\alpha, \\
c_{\ell^{\prime}}(u)+\alpha=\gamma+\alpha\end{array}$ & $\ell\left(P_{1}\right)=(\alpha, \beta, \beta, \gamma, \alpha)$ & Since $c_{\ell^{\prime}}(u) \neq c_{\ell^{\prime}}(v)$, then $c_{\ell^{\prime}}(v)+\alpha \neq \gamma+\alpha$ \\
\hline $\begin{array}{l}c_{\ell^{\prime}}(u)+\alpha \neq c_{\ell^{\prime}}\left(u^{\prime}\right), \\
c_{\ell^{\prime}}(v)+\alpha \neq c_{\ell^{\prime}}\left(v^{\prime}\right), \\
c_{\ell^{\prime}}(v)+\alpha=\beta+\alpha \\
\end{array}$ & $\ell\left(P_{1}\right)=(\alpha, \beta, \beta, \gamma, \alpha)$ & $\begin{array}{c}\text { Since } c_{\ell^{\prime}}(u) \neq c_{\ell^{\prime}}(v) \text {, then } \\
c_{\ell^{\prime}}(u)+\alpha \neq \beta+\alpha\end{array}$ \\
\hline $\begin{array}{l}c_{\ell^{\prime}}(u)+\alpha=c_{\ell^{\prime}}\left(u^{\prime}\right), \\
c_{\ell^{\prime}}(v)+\beta \neq c_{\ell^{\prime}}\left(v^{\prime}\right), \\
c_{\ell^{\prime}}(v)+\beta \neq \beta+\gamma \\
c_{\ell^{\prime}}(u)+\beta \neq \beta+\alpha\end{array}$ & $\ell\left(P_{1}\right)=(\beta, \alpha, \alpha, \gamma, \beta)$ & By the conditions of the subcase. \\
\hline $\begin{array}{l}c_{\ell^{\prime}}(u)+\alpha=c_{\ell^{\prime}}\left(u^{\prime}\right), \\
c_{\ell^{\prime}}(v)+\beta \neq c_{\ell^{\prime}}\left(v^{\prime}\right), \\
c_{\ell^{\prime}}(v)+\beta \neq \beta+\gamma, \\
c_{\ell^{\prime}}(u)+\beta=\beta+\alpha\end{array}$ & $\ell\left(P_{1}\right)=(\beta, \gamma, \alpha, \alpha, \beta)$ & $\begin{array}{c}\text { Since } c_{\ell^{\prime}}(u) \neq c_{\ell^{\prime}}(v) \text {, then } \\
c_{\ell^{\prime}}(v)+\beta \neq \beta+\alpha .\end{array}$ \\
\hline $\begin{array}{c}c_{\ell^{\prime}}(u)+\alpha=c_{\ell^{\prime}}\left(u^{\prime}\right), \\
c_{\ell^{\prime}}(v)+\beta \neq c_{\ell^{\prime}}\left(v^{\prime}\right), \\
c_{\ell^{\prime}}(v)+\beta=\beta+\gamma \\
\end{array}$ & $\ell\left(P_{1}\right)=(\beta, \gamma, \alpha, \alpha, \beta)$ & $\begin{array}{c}\text { Since } c_{\ell^{\prime}}(u) \neq c_{\ell^{\prime}}(v) \text {, then } \\
c_{\ell^{\prime}}(u)+\beta \neq \beta+\gamma .\end{array}$ \\
\hline $\begin{array}{c}c_{\ell^{\prime}}(u)+\alpha=c_{\ell^{\prime}}\left(u^{\prime}\right) \\
c_{\ell^{\prime}}(v)+\beta=c_{\ell^{\prime}}\left(v^{\prime}\right) \\
c_{\ell^{\prime}}(u)+\beta=c_{\ell^{\prime}}(v)+\alpha \\
c_{\ell^{\prime}}(v)+\gamma \neq \gamma+\alpha \\
c_{\ell^{\prime}}(u)+\beta \neq 2 \beta \\
\end{array}$ & $\ell\left(P_{1}\right)=(\beta, \beta, \alpha, \alpha, \gamma)$ & $\begin{array}{l}\text { By the conditions of the subcase. } \\
\text { Note also that } c_{\ell}(u) \neq c_{\ell}(v)\end{array}$ \\
\hline $\begin{array}{c}c_{\ell^{\prime}}(u)+\alpha=c_{\ell^{\prime}}\left(u^{\prime}\right) \\
c_{\ell^{\prime}}(v)+\beta=c_{\ell^{\prime}}\left(v^{\prime}\right) \\
c_{\ell^{\prime}}(u)+\beta=c_{\ell^{\prime}}(v)+\alpha \\
c_{\ell^{\prime}}(v)+\gamma \neq \gamma+\alpha \\
c_{\ell^{\prime}}(u)+\beta=2 \beta\end{array}$ & $\ell\left(P_{1}\right)=(\gamma, \alpha, \beta, \beta, \alpha)$ & $\begin{array}{c}\text { Since } c_{\ell^{\prime}}(u)=\beta, \text { then } \\
c_{\ell^{\prime}}(v)=2 \beta-\alpha, c_{\ell^{\prime}}\left(u^{\prime}\right)=\beta+\alpha, \\
c_{\ell^{\prime}}\left(v^{\prime}\right)=3 \beta-\alpha, c_{\ell}(u)=\beta+\gamma, \text { and } \\
c_{\ell}(v)=2 \beta . \text { It can then be verified } \\
\text { that there are no conflicts. }\end{array}$ \\
\hline $\begin{array}{c}c_{\ell^{\prime}}(u)+\alpha=c_{\ell^{\prime}}\left(u^{\prime}\right), \\
c_{\ell^{\prime}}(v)+\beta=c_{\ell^{\prime}}\left(v^{\prime}\right) \\
c_{\ell^{\prime}}(u)+\beta=c_{\ell^{\prime}}(v)+\alpha \\
c_{\ell^{\prime}}(v)+\gamma=\gamma+\alpha\end{array}$ & $\ell\left(P_{1}\right)=(\beta, \alpha, \alpha, \beta, \gamma)$ & $\begin{array}{c}\text { By the last two conditions of the } \\
\text { subcase, } c_{\ell^{\prime}}(u)+\beta=2 \alpha \\
\text { Thus, } c_{\ell}(u) \neq \beta+\alpha \\
\text { Note also that } c_{\ell}(u) \neq c_{\ell}(v)\end{array}$ \\
\hline $\begin{array}{c}c_{\ell^{\prime}}(u)+\alpha=c_{\ell^{\prime}}\left(u^{\prime}\right) \\
c_{\ell^{\prime}}(v)+\beta=c_{\ell^{\prime}}\left(v^{\prime}\right) \\
c_{\ell^{\prime}}(u)+\beta \neq c_{\ell^{\prime}}(v)+\alpha \\
c_{\ell^{\prime}}(v)+\alpha \neq 2 \alpha \\
c_{\ell^{\prime}}(u)+\beta \neq 2 \beta \\
\end{array}$ & $\ell\left(P_{1}\right)=(\beta, \beta, \gamma, \alpha, \alpha)$ & $\begin{array}{l}\text { By the conditions of the subcase. } \\
\text { Note also that } c_{\ell}(u) \neq c_{\ell}(v)\end{array}$ \\
\hline $\begin{array}{c}c_{\ell^{\prime}}(u)+\alpha=c_{\ell^{\prime}}\left(u^{\prime}\right) \\
c_{\ell^{\prime}}(v)+\beta=c_{\ell^{\prime}}\left(v^{\prime}\right) \\
c_{\ell^{\prime}}(u)+\beta \neq c_{\ell^{\prime}}(v)+\alpha \\
c_{\ell^{\prime}}(v)+\alpha \neq 2 \alpha \\
c_{\ell^{\prime}}(u)+\beta=2 \beta\end{array}$ & $\ell\left(P_{1}\right)=(\beta, \alpha, \gamma, \beta, \alpha)$ & $\begin{array}{c}\text { Note that } \beta \geq 2 \text { since } c_{\ell^{\prime}}(u)=\beta \text {. } \\
\text { If } \beta=2: c_{\ell}(u)=4, c_{\ell}(v)=5, c_{\ell}\left(v^{\prime}\right)=6 \& \\
c_{\ell}\left(u^{\prime}\right)=c_{\ell}(x)=c_{\ell}(y)=3 \\
\text { If } \beta=3: c_{\ell}(u)=6, c_{\ell}(v)=4, c_{\ell}\left(v^{\prime}\right)=7 \& \\
c_{\ell}\left(u^{\prime}\right)=c_{\ell}(x)=c_{\ell}(y)=5\end{array}$ \\
\hline $\begin{array}{c}c_{\ell^{\prime}}(u)+\alpha=c_{\ell^{\prime}}\left(u^{\prime}\right) \\
c_{\ell^{\prime}}(v)+\beta=c_{\ell^{\prime}}\left(v^{\prime}\right) \\
c_{\ell^{\prime}}(u)+\beta \neq c_{\ell^{\prime}}(v)+\alpha \\
c_{\ell^{\prime}}(v)+\alpha=2 \alpha \\
c_{\ell^{\prime}}(u)+\beta \neq \beta+\alpha\end{array}$ & $\ell\left(P_{1}\right)=(\beta, \alpha, \gamma, \beta, \alpha)$ & $\begin{array}{l}\text { By the conditions of the subcase. } \\
\text { Note also that } c_{\ell}(u) \neq c_{\ell}(v)\end{array}$ \\
\hline
\end{tabular}

Table 2: The 12 subcases for $p=1$. 


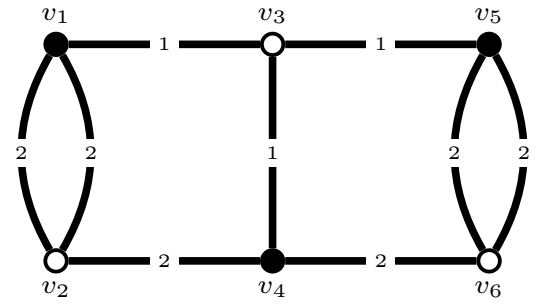

(a) With parallel edges.

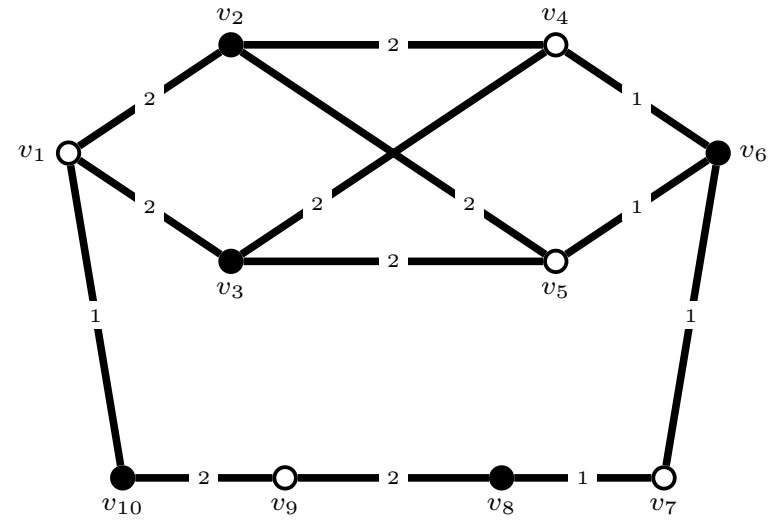

(b) With adjacent degree-2 vertices.

Figure 6: Proper 2-labellings of two bipartite graphs $G$ with $2=\chi_{\Sigma}(G)<\overline{\chi \Sigma}(G)=3$.

$\alpha \in\{1,2\}$, and that $\ell^{\prime}(w x)=\beta$ for some $\beta \in\{1,2\}$. Set $\{\bar{\alpha}\}=\{1,2\} \backslash\{\alpha\}$ and $\{\bar{\beta}\}=\{1,2\} \backslash\{\beta\}$. Then, by Observation 2.1, we have $\ell^{\prime}(x y)=\bar{\alpha}, \ell^{\prime}(y z)=\bar{\beta}$, and $\ell^{\prime}(z v)=\alpha$. By the properness of $\ell^{\prime}$, since $u$ and $v$ are adjacent in $G^{\prime}$, we have that $c_{\ell^{\prime}}(u) \neq c_{\ell^{\prime}}(v)$. This implies that the 2-labelling $\ell$ of $G$ obtained from $\ell^{\prime}$ by assigning label $\alpha$ to the edge $u v$ that was subdivided for constructing $G^{\prime}$, and setting $\ell(e)=\ell^{\prime}(e)$ for every $e \in E(G) \cap E\left(G^{\prime}\right)$, is proper. Furthermore, we have $\left\{\ell^{\prime}(w x), \ell^{\prime}(y z)\right\}=$ $\left\{\ell^{\prime}(x y), \ell^{\prime}(z v)\right\}=\{1,2\}$. Hence, $\mathrm{nb}_{\ell}(1)=\mathrm{nb}_{\ell^{\prime}}(1)-2$ and $\mathrm{nb}_{\ell}(2)=\mathrm{nb}_{\ell^{\prime}}(2)-2$. So, $\ell$ is an equitable proper 2-labelling of $G$, a contradiction. Thus, $\overline{\chi_{\Sigma}}\left(G^{\prime}\right) \geq 3$.

Theorem 3.4 (Operation 2). Let $G$ be a graph with $\overline{\chi_{\Sigma}}(G) \geq 3$. If $G$ has an edge uv with $d(u)=$ $d(v)=2$, then the graph $G^{\prime}$ obtained from $G$ by subdividing uv four times verifies $\overline{\chi_{\Sigma}}\left(G^{\prime}\right) \geq 3$.

Proof. Let us denote by $(u, w, x, y, z, v)$ the path of length 5 of $G^{\prime}$ that results from the subdivision of $u v$. Let us denote the other neighbour of $u$ by $u^{\prime}$, and the other neighbour of $v$ by $v^{\prime}$. Assume there exists $\ell^{\prime}$, an equitable proper 2-labelling of $G^{\prime}$. Assume that $\ell^{\prime}\left(u^{\prime} u\right)=\alpha$ for some $\alpha \in\{1,2\}$, and that $\ell^{\prime}(u w)=\beta$ for some $\beta \in\{1,2\}$. Set $\{\bar{\alpha}\}=\{1,2\} \backslash\{\alpha\}$ and $\{\bar{\beta}\}=\{1,2\} \backslash\{\beta\}$. According to Observation 2.1, we have $\ell^{\prime}(w x)=\bar{\alpha}, \ell^{\prime}(x y)=\bar{\beta}, \ell^{\prime}(y z)=\alpha, \ell^{\prime}(z v)=\beta$, and $\ell^{\prime}\left(v v^{\prime}\right)=\bar{\alpha}$. It follows that the 2-labelling $\ell$ of $G$ obtained from $\ell^{\prime}$ by setting $\ell(u v)=\beta$ and $\ell(e)=\ell^{\prime}(e)$ for every $e \in E(G) \cap E\left(G^{\prime}\right)$ is proper. In particular, note that the above implies that $c_{\ell}(u)=c_{\ell^{\prime}}(u) \neq c_{\ell^{\prime}}(v)=c_{\ell}(v)$, although $u$ and $v$ are not adjacent in $G^{\prime}$. Furthermore, we have $\left\{\ell^{\prime}(w x), \ell^{\prime}(y z)\right\}=\left\{\ell^{\prime}(x y), \ell^{\prime}(z v)\right\}=\{1,2\}$. This implies that $\mathrm{nb}_{\ell}(1)=\mathrm{nb}_{\ell^{\prime}}(1)-2$ and $\mathrm{nb}_{\ell}(2)=\mathrm{nb}_{\ell^{\prime}}(2)-2$. So, $\ell$ is an equitable proper 2-labelling of $G$, a contradiction. Thus, $\overline{\chi_{\Sigma}}\left(G^{\prime}\right) \geq 3$.

We note in particular that Operations 1 and 2 mentioned in Theorems 3.3 and 3.4, when performed on bipartite graphs, yield graphs that are also bipartite. From this observation, we come up with two infinite families of bipartite graphs $G$ verifying $2=\chi_{\Sigma}(G)<\overline{\chi_{\Sigma}}(G)=3$.

The first such family is obtained by repeatedly applying Operations 1 and 2 from the cubic bipartite multigraph depicted in Figure 6(a). This graph indeed has the following properties:

Theorem 3.5. Let $G$ be the cubic bipartite multigraph depicted in Figure $6(a)$. Then, $\chi_{\Sigma}(G)=2$ and $\overline{\chi \Sigma}(G)=3$.

Proof. Since $G$ is cubic, we have $\chi_{\Sigma}(G)>1$ and thus, $\overline{\chi_{\Sigma}}(G)>1$. Actually, we even have $\chi_{\Sigma}(G)=$ 2 since $G$ does not match the definition of an odd multi-cactus (a proper 2-labelling is also included in Figure 6(a)). Towards a contradiction, assume $G$ admits an equitable proper 2-labelling $\ell$. In 
order to have $c_{\ell}\left(v_{1}\right) \neq c_{\ell}\left(v_{2}\right)$, we have $\ell\left(v_{1} v_{3}\right) \neq \ell\left(v_{2} v_{4}\right)$. Similarly, since $c_{\ell}\left(v_{5}\right) \neq c_{\ell}\left(v_{6}\right)$, we have $\ell\left(v_{5} v_{3}\right) \neq \ell\left(v_{6} v_{4}\right)$. Now, since $c_{\ell}\left(v_{3}\right) \neq c_{\ell}\left(v_{4}\right)$, we have $\ell\left(v_{1} v_{3}\right)=\ell\left(v_{3} v_{5}\right)$ and $\ell\left(v_{2} v_{4}\right)=\ell\left(v_{4} v_{6}\right)$, since otherwise, $c_{\ell}\left(v_{3}\right)=c_{\ell}\left(v_{4}\right)=1+2+\ell\left(v_{3} v_{4}\right)$ by the previous argument. Thus, without loss of generality, we may assume that $\ell\left(v_{1} v_{3}\right)=\ell\left(v_{3} v_{5}\right)=1$ and $\ell\left(v_{2} v_{4}\right)=\ell\left(v_{4} v_{6}\right)=2$.

Assume now that $\ell\left(v_{3} v_{4}\right)=1$. This gives $c_{\ell}\left(v_{3}\right)=3$ and $c_{\ell}\left(v_{4}\right)=5$. Now, note that the two edges joining $v_{1}$ and $v_{2}$, and similarly the two edges joining $v_{5}$ and $v_{6}$, cannot both be assigned label 1 (as, otherwise, $v_{1}$ or $v_{5}$ would be in conflict with $v_{3}$ ). Similarly, to avoid a conflict with $v_{4}$, the two edges joining $v_{1}$ and $v_{2}$, and similarly the two edges joining $v_{5}$ and $v_{6}$, cannot be assigned labels 1 and 2 respectively. Thus, these four edges must be assigned label 2, which means that $\mathrm{nb}_{\ell}(1)=3$ and $\mathrm{nb}_{\ell}(2)=6$. This is a contradiction to the equitability of $\ell$. Similar arguments can be used to show that we cannot have $\ell\left(v_{3} v_{4}\right)=2$ either. Thus, $\overline{\chi \Sigma}(G)>2$, and one can easily come up with equitable proper 3-labellings of $G$.

A second infinite family of bipartite graphs $G$ with $2=\chi_{\Sigma}(G)<\overline{\chi_{\Sigma}}(G)=3$ is obtained by repeatedly applying Operation 2, described in Theorem 3.4, to the graph depicted in Figure 6(b) (note that this is $K_{3,3}$ with one edge subdivided 4 times), which indeed has the following properties:

Theorem 3.6. Let $G$ be the subcubic bipartite graph depicted in Figure $6(b)$. Then, $\chi_{\Sigma}(G)=2$ and $\overline{\chi_{\Sigma}}(G)=3$.

Proof. Again, $G$ is not locally irregular (i.e., it has adjacent vertices with the same degree) and does not match the structure of an odd multi-cactus, so $\chi_{\Sigma}(G)=2$ (a proper 2-labelling is also included in Figure 6(b)). We now prove that $\overline{\chi_{\Sigma}}(G)=3$. Let us suppose that there exists an equitable proper 2-labelling $\ell$ of $G$. By Observation 2.1, we must have $\ell\left(v_{1} v_{10}\right) \neq \ell\left(v_{9} v_{8}\right)$ and $\ell\left(v_{7} v_{6}\right) \neq \ell\left(v_{9} v_{8}\right)$, and thus, $\ell\left(v_{1} v_{10}\right)=\ell\left(v_{7} v_{6}\right)$. Moreover, either $\ell\left(v_{10} v_{9}\right)=\ell\left(v_{1} v_{10}\right)=\ell\left(v_{7} v_{6}\right)$ and $\ell\left(v_{9} v_{8}\right)=\ell\left(v_{8} v_{7}\right)$ or $\ell\left(v_{1} v_{10}\right)=\ell\left(v_{8} v_{7}\right)=\ell\left(v_{7} v_{6}\right)$ and $\ell\left(v_{10} v_{9}\right)=\ell\left(v_{9} v_{8}\right)$. This implies that each of the labels 1 and 2 appears exactly twice in the edges $v_{10} v_{9}, v_{9} v_{8}, v_{8} v_{7}, v_{7} v_{6}$.

Let $G^{\prime}$ be the graph obtained from $G$ by replacing the path $P=\left(v_{1}, v_{10}, v_{9}, v_{8}, v_{7}, v_{6}\right)$ by a single edge $v_{1} v_{6}$. Moreover, let $\ell^{\prime}$ be the labelling of $G^{\prime}$ such that $\ell^{\prime}(e)=\ell(e)$ for every edge $e \in E\left(G^{\prime}\right) \cap E(G)$, and $\ell^{\prime}\left(v_{1} v_{6}\right)=\ell\left(v_{1} v_{10}\right)$. Since $\ell$ is equitable and due to the previous remark, $\ell^{\prime}$ is an equitable 2-labelling of $G$. Now, it suffices to show that $\ell^{\prime}$ is also proper. If this is the case, we arrive at a contradiction since $G^{\prime}$ is isomorphic to $K_{3,3}$ and $\overline{\chi_{\Sigma}}\left(K_{3,3}\right)=3$ (as proved in [1]).

For the sake of contradiction, suppose that $\ell^{\prime}$ is not proper. Since $\ell$ is a proper labelling of $G$, it follows that $c_{\ell^{\prime}}\left(v_{1}\right)=c_{\ell^{\prime}}\left(v_{6}\right)$ in $G^{\prime}$ and that these are the only two vertices that are in conflict. Observe that $G^{\prime}$ is a cubic graph and thus, for each $v \in V\left(G^{\prime}\right)$, we have $c_{\ell^{\prime}}(v) \in\{3,4,5,6\}$. We distinguish the following cases:

- $c_{\ell^{\prime}}\left(v_{1}\right)=c_{\ell^{\prime}}\left(v_{6}\right)=3$.

In this case, $\ell^{\prime}\left(v_{4} v_{6}\right)=\ell^{\prime}\left(v_{2} v_{1}\right)=\ell^{\prime}\left(v_{3} v_{1}\right)=\ell^{\prime}\left(v_{5} v_{6}\right)=1$, and so, $c_{\ell^{\prime}}\left(v_{2}\right), c_{\ell^{\prime}}\left(v_{3}\right), c_{\ell^{\prime}}\left(v_{4}\right)$, $c_{\ell^{\prime}}\left(v_{5}\right) \neq 6$. Moreover, so that $3=c_{\ell^{\prime}}\left(v_{1}\right) \notin\left\{c_{\ell^{\prime}}\left(v_{2}\right), c_{\ell^{\prime}}\left(v_{3}\right)\right\}$ and $3=c_{\ell^{\prime}}\left(v_{6}\right) \notin\left\{c_{\ell^{\prime}}\left(v_{4}\right)\right.$, $\left.c_{\ell^{\prime}}\left(v_{5}\right)\right\}$, we have that $c_{\ell^{\prime}}\left(v_{2}\right), c_{\ell^{\prime}}\left(v_{3}\right), c_{\ell^{\prime}}\left(v_{4}\right), c_{\ell^{\prime}}\left(v_{5}\right) \in\{4,5\}$. By symmetry, let us assume that $c_{\ell^{\prime}}\left(v_{2}\right)=5$. Then, we must have $c_{\ell^{\prime}}\left(v_{4}\right)=c_{\ell^{\prime}}\left(v_{5}\right)=4$ which means $\ell^{\prime}\left(v_{3} v_{4}\right)=1$ and that we must have $c_{\ell^{\prime}}\left(v_{3}\right)=5$, which is impossible since $\ell^{\prime}\left(v_{3} v_{4}\right)=1$.

- $c_{\ell^{\prime}}\left(v_{1}\right)=c_{\ell^{\prime}}\left(v_{6}\right)=4$.

First, let us assume that $\ell^{\prime}\left(v_{1} v_{6}\right)=2$. In this case, $\ell^{\prime}\left(v_{4} v_{6}\right)=\ell^{\prime}\left(v_{2} v_{1}\right)=\ell^{\prime}\left(v_{3} v_{1}\right)=\ell^{\prime}\left(v_{5} v_{6}\right)=$ 1 , and so, $c_{\ell^{\prime}}\left(v_{2}\right), c_{\ell^{\prime}}\left(v_{3}\right), c_{\ell^{\prime}}\left(v_{4}\right), c_{\ell^{\prime}}\left(v_{5}\right) \neq 6$. Moreover, so that $4=c_{\ell^{\prime}}\left(v_{1}\right) \notin\left\{c_{\ell^{\prime}}\left(v_{2}\right), c_{\ell^{\prime}}\left(v_{3}\right)\right\}$ and $4=c_{\ell^{\prime}}\left(v_{6}\right) \notin\left\{c_{\ell^{\prime}}\left(v_{4}\right), c_{\ell^{\prime}}\left(v_{5}\right)\right\}$, we have that $c_{\ell^{\prime}}\left(v_{2}\right), c_{\ell^{\prime}}\left(v_{3}\right), c_{\ell^{\prime}}\left(v_{4}\right), c_{\ell^{\prime}}\left(v_{5}\right) \in\{3,5\}$. By symmetry, let us assume that $c_{\ell^{\prime}}\left(v_{2}\right)=5$. Then, we have $c_{\ell^{\prime}}\left(v_{4}\right) \in\{4,5\}$, which conflicts with either $v_{2}$ or $v_{6}$.

Second, let us assume that $\ell^{\prime}\left(v_{1} v_{6}\right)=1$. In this case, we may assume by symmetry that $\ell^{\prime}\left(v_{4} v_{6}\right)=\ell^{\prime}\left(v_{2} v_{1}\right)=1$ and $\ell^{\prime}\left(v_{3} v_{1}\right)=\ell^{\prime}\left(v_{5} v_{6}\right)=2$, and so, $c_{\ell^{\prime}}\left(v_{2}\right), c_{\ell^{\prime}}\left(v_{4}\right) \in\{3,5\}$. By 


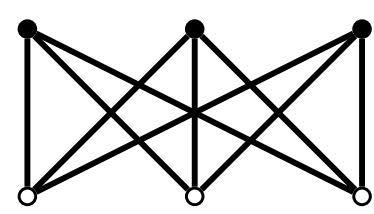

(a)

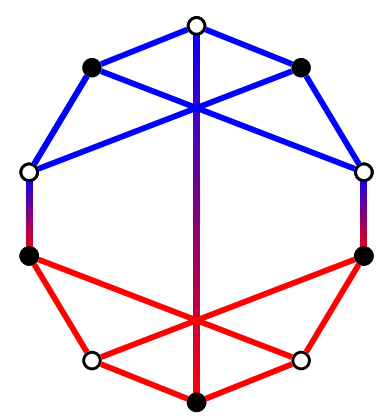

(c)

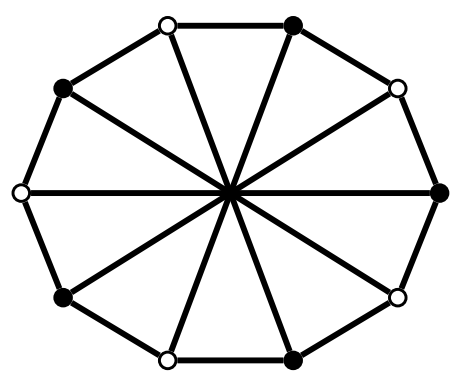

(b)

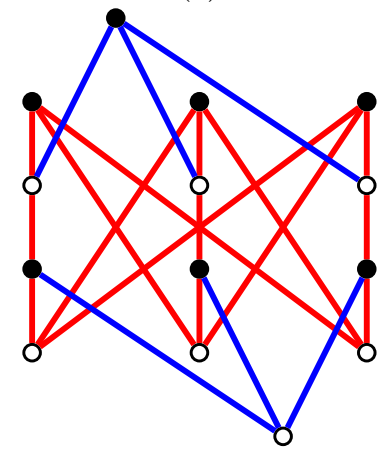

(d)

Figure 7: Four cubic bipartite graphs $G$ with $\overline{\chi_{\Sigma}}(G)=3$.

symmetry, let us assume that $c_{\ell^{\prime}}\left(v_{2}\right)=5$. Therefore, $\ell^{\prime}\left(v_{2} v_{4}\right)=2$ and $c_{\ell^{\prime}}\left(v_{4}\right) \in\{4,5\}$ which conflicts either with $v_{2}$ or $v_{6}$.

- $c_{\ell^{\prime}}\left(v_{1}\right)=c_{\ell^{\prime}}\left(v_{6}\right) \in\{5,6\}$.

These cases can be proved similarly to the previous cases by switching labels 1 and 2 .

Corollary 3.7. There exist infinitely many bipartite graphs $G$ with $2=\chi_{\Sigma}(G)<\overline{\chi_{\Sigma}}(G)=3$.

We ran computer programs to come up with examples of bipartite graphs admitting no equitable proper 2-labellings. In particular, we were able to check all cubic bipartite graphs with up to 25 vertices, and all subcubic (non-cubic) bipartite graphs with up to 17 vertices. It turns out that, for this restricted sampling, all bipartite graphs $G$ with $2=\chi_{\Sigma}(G)<\overline{\chi_{\Sigma}}(G)=3$ are either small, or modifications of these graphs obtained through Operations 1 and 2 described earlier.

All these graphs share particular properties of interest. They are all of minimum degree at least 2 and of maximum degree 3. They also share two important properties of odd multi-cacti, as they are 2-connected and both their partition classes are of odd size. All of these graphs are also related to $K_{3,3}$ somehow. For instance, the graph in Figure $7(\mathrm{c})$ can be obtained by "gluing" two $K_{3,3}$ 's one (with red edges) onto the other (with blue edges) (note indeed that by contracting the bottom five vertices to a single vertex (and similarly for the top five vertices), it results in $K_{3,3}$ ). Also, the graph in Figure $7(\mathrm{~d})$ can be obtained by subdividing twice the edges of a perfect matching of $K_{3,3}$ (resulting in the red edges) and joining the resulting vertices to two new degree-3 vertices (via blue edges). In the non-cubic case, we observed that repeatedly applying Operation 1 on the edges of a perfect matching of $K_{3,3}$ yields subcubic bipartite graphs $G$ with $2=\chi_{\Sigma}(G)<\overline{\chi_{\Sigma}}(G)=3$.

It might be that the four graphs (already mentioned in [11]) depicted in Figure 7 are the only cubic bipartite graphs $G$ with $\chi_{\Sigma}(G)=2$ and $\overline{\chi_{\Sigma}}(G)=3$. Our feeling is that as soon as a cubic 
bipartite graph is large enough, there should always be several ways to locally alter a proper 2labelling to make it equitable. As a result, we were unsuccessful in coming up with infinite families of such graphs. For instance, a natural way for generalising the graphs in Figures $7(\mathrm{a})$ and $7(\mathrm{~b})$ is as follows: for any $n \equiv 2 \bmod 4$, we denote by $C_{n}^{\times}$the cubic graph obtained from $C_{n}$ by adding an edge between any two antipodal vertices. Note indeed that the graph in Figure $7(\mathrm{a})$, which is $K_{3,3}$, is also $C_{6}^{\times}$, while the graph in Figure $7(\mathrm{~b})$ is $C_{10}^{\times}$. Our experimentations show that, unfortunately, it seems that $\overline{\chi_{\Sigma}}\left(C_{n}^{\times}\right)=2$ whenever $n \geq 14$.

In light of these arguments, let us finish this section by raising the following questions:

Question 3.8. Let $G$ be a bipartite graph with $2=\chi_{\Sigma}(G)<\overline{\chi_{\Sigma}}(G)=3$.

- Can we have $\delta(G)=1$ ?

- Can we have $\Delta(G) \geq 4$ ?

- Can $G$ have cut vertices?

- Can we have $|V(G)| \not \equiv 2 \bmod 4$ ?

- Can $G$ be cubic with $|V(G)|$ being arbitrarily large?

- Was $G$ obtained from $K_{3,3}$ by repeatedly applying certain operations?

\section{Equitable proper labellings of regular bipartite graphs}

In this section, we mainly prove that, for any $k \geq 3$, every $k$-regular bipartite graph admits equitable proper $k$-labellings. As a particular case, we get that cubic bipartite graphs form another family of graphs verifying Conjecture 1.2.

Our proof makes use of the following result of König from 1916, which says that regular bipartite graphs are class 1 . Recall that a proper edge-colouring of a graph is an edge-colouring where no two adjacent edges get assigned the same colour. In other words, the edge-set of any $k$-regular bipartite graph can be partitioned into $k$ perfect matchings.

Lemma 4.1 (König [7]). All k-regular bipartite graphs admit proper $k$-edge-colourings.

We are now ready for our main result.

Theorem 4.2. For all $k \geq 3$, if $G=(A, B, E)$ is a k-regular bipartite graph, then $\overline{\chi \Sigma}(G) \leq k$.

Proof. Initially, apply a proper $k$-edge colouring to $G$, which exists by Lemma 4.1 . This initial $k$-labelling $\ell$ is equitable, but it is not proper, since every vertex has colour $p=\frac{k(k+1)}{2}$ (each vertex is incident to exactly $k$ edges, each with a unique label from 1 to $k$ ). The following fourstep algorithm which makes local swaps of labels is applied to the $k$-labelling $\ell$ of $G$, until the $k$-labelling $\ell$ becomes proper. Note that the algorithm only swaps labels of edges, and therefore, the $k$-labelling $\ell$ remains equitable throughout. In what follows, for a vertex $v \in V(G)$, an edge $u v \in E(G)$, and an integer $i \geq 1$, let $c_{\ell}^{i}(v)$ and $\ell^{i}(u v)$ be the colour of the vertex $v$ and the label of the edge $u v$ respectively, after the $(i-1)^{t h}$ iteration (and before the $i^{t h}$ iteration) of the current step being considered. Also, the superscript is omitted from the colour notation when the current colour is being mentioned. The algorithm begins as follows:

1. While there exists a subgraph of $G$ isomorphic to $P_{3}=(u, x, v)$ such that $u, v \in A$ and $c_{\ell}(u)=c_{\ell}(v)=p$, swap $\ell(u x)$ with $\ell(v x)$.

There are three things to note after the first step of the algorithm. The first is that, for each pair of vertices $u, v \in A$ that are dealt with in the $i^{t h}$ iteration of Step $1, c_{\ell}^{i+1}(u) \neq p$ and $c_{\ell}^{i+1}(v) \neq p$ for all $i \geq 1$. Indeed, their colours were $p$ before the step was executed but since $\ell(u x) \neq \ell(v x)$ 
for all these pairs $u, v \in A$ ( $x$ is incident to exactly one edge with label $j$ for all $1 \leq j \leq k$ ), their colours cannot be $p$ after the step is executed. Also, only the labels of edges incident to the vertices $u$ and $v$ are changed and so, the edges whose labels are changed at each execution of Step 1 are all disjoint. The second thing is that, for every vertex $u \in B$, we have $c_{\ell}(u)=p$. The third thing is that, once Step 1 can no longer be executed, for any two vertices $u, v \in A$ such that $c_{\ell}(u)=c_{\ell}(v)=p$, we have that $\operatorname{dist}(u, v) \geq 4$. Now, the algorithm proceeds as follows:

2. While there exists an induced subgraph of $G$ isomorphic to $P_{5}=(u, x, z, y, v)$ such that $u, v \in A$ and $c_{\ell}(u)=c_{\ell}(v)=p$,

(a) swap $\ell(u x)$ with $\ell(x z)$ if this results in $c_{\ell}(z) \neq p$;

(b) else, swap $\ell(v y)$ with $\ell(y z)$ if this results in $c_{\ell}(z) \neq p$;

(c) else, swap $\ell(u x)$ with $\ell(x z)$ and $\ell(v y)$ with $\ell(y z)$.

Claim 4.3. If one of Steps 2(a)-(c) is executed on the $i^{\text {th }}$ iteration of Step 2, then $c_{\ell}^{i+1}(z) \neq p$. Moreover, after any of them is executed, each vertex in $B$ is still incident to exactly one edge with label $j$ for all $1 \leq j \leq k$.

Proof of the claim. If Step 2(a) or Step 2(b) is executed, then $c_{\ell}^{i+1}(z) \neq p$ by definition. If Step 2 (a) cannot be executed, then $c_{\ell}^{i}(z)-\ell^{i}(x z)+\ell^{i}(u x)=p$. Observe that $\ell^{i}(u x) \neq \ell^{i}(x z)$ and $\ell^{i}(v y) \neq \ell^{i}(y z)$ since $x, y \in B$ and each vertex in $B$ is still incident to exactly one edge with label $j$ for all $1 \leq j \leq k$ (trivial induction on the number of times such a process has been performed before this step). Therefore, $c_{\ell}^{i}(z)-\ell^{i}(x z)+\ell^{i}(u x)-\ell^{i}(y z)+\ell^{i}(v y)=p-\ell^{i}(y z)+\ell^{i}(v y) \neq p$ and so, $c_{\ell}^{i+1}(z) \neq p$ if Step 2(c) is executed.

Note that in all cases of Step 2, after its $i^{\text {th }}$ execution, either $c_{\ell}^{i+1}(u) \neq p$ or $c_{\ell}^{i+1}(v) \neq p$. Moreover, if $c_{\ell}^{i+1}(u)=p\left(c_{\ell}^{i+1}(v)=p\right.$ respectively), then none of the labels of the edges incident to $u$ ( $v$ respectively) were changed. Note that Step 2 eventually ends since no new vertices get colour $p$ by Claim 4.3 and at least one vertex changes from colour $p$ to another colour after each execution of Step 2. Once Step 2 can no longer be executed, for any two vertices $u, v \in A$ such that $c_{\ell}(u)=c_{\ell}(v)=p$, we have that $\operatorname{dist}(u, v)>4$. The algorithm proceeds as follows:

3. While there exists a subgraph of $G$ isomorphic to $C_{4}=(u, x, z, y, u)$ such that $u \in A$ and $c_{\ell}(u)=p$

(a) swap $\ell(u x)$ with $\ell(x z)$ if this results in $c_{\ell}(z) \neq p$;

(b) else, swap $\ell(u y)$ with $\ell(y z)$ if this results in $c_{\ell}(z) \neq p$;

(c) else, swap $\ell(u x)$ with $\ell(x z)$ and $\ell(u y)$ with $\ell(y z)$.

From a proof analogous to that of Claim 4.3, if one of Steps 3(a)-(c) is executed at the $i^{t h}$ iteration of Step 3, then $c_{\ell}^{i+1}(z) \neq p$. Furthermore, in all cases of Step 3, after its $i^{\text {th }}$ iteration, we have $c_{\ell}^{i+1}(u) \neq p$, which is obvious except for in the case that Step 3(c) was executed. Note that if $c_{\ell}^{i+1}(u)=p$ after the $i^{t h}$ iteration of Step $3(\mathrm{c})$, then $c_{\ell}^{i}(u)-\ell^{i}(u x)+\ell^{i}(x z)-\ell^{i}(u y)+\ell^{i}(y z)=p$. In this case, $\ell^{i}(x z)-\ell^{i}(u x)=\ell^{i}(u y)-\ell^{i}(y z)$, since $c_{\ell}^{i}(u)=p$. But then, since Steps 3(a) and $3(\mathrm{~b})$ were not executable, $c_{\ell}^{i}(z)-\ell^{i}(x z)+\ell^{i}(u x)=c_{\ell}^{i}(z)-\ell^{i}(y z)+\ell^{i}(u y)=p$, which implies that $\ell^{i}(u x)-\ell^{i}(x z)=\ell^{i}(u y)-\ell^{i}(y z)$. This is a contradiction since both $\ell^{i}(x z)-\ell^{i}(u x)=\ell^{i}(u y)-\ell^{i}(y z)$ and $\ell^{i}(u x)-\ell^{i}(x z)=\ell^{i}(u y)-\ell^{i}(y z)$ hold if and only if $\ell^{i}(x z)=\ell^{i}(u x)$, but $\ell^{i}(x z) \neq \ell^{i}(u x)$ since $x, y \in B$ and each of the vertices of $B$ is still incident to exactly one edge with label $j$ for all $1 \leq j \leq k$, even after each iteration of Step 3 is executed until Step 3 can no longer be executed. Therefore, Step 3(a) or 3(b) was executable and so, Step 3(c) would not have been executed.

Note that Step 3 eventually ends since no new vertices get colour $p$ and one vertex changes from colour $p$ to another colour after each execution of Step 3. Once Step 3 can no longer be executed, then for any vertex $u \in A$ such that $c_{\ell}(u)=p$, we have that for any two vertices $x, y \in N(u)$, it holds that $N(x) \cap N(y)=u$. The remainder of the algorithm depends on the value of $k$ with the 
case where $k=3$ being different from the case $k \geq 4$. In what follows, we denote by $S_{x}$ the star with $x$ leaves (being isomorphic to $K_{1, x}$ ).

Case $k=3$ : note that $p=6$ in this case. The algorithm proceeds as follows:

4. While there exists a subgraph of $G$ isomorphic to $S_{3}$, with center $u$ and leaves $x, y, z$ such that $u \in A$ and for all $w \in N(u)$, we have that $c_{\ell}(u)=c_{\ell}(w)=6$, then, w.l.o.g., we may assume that $\ell^{i}(u x)=1, \ell^{i}(u y)=2$, and $\ell^{i}(u z)=3$, and

(a) if for some $w \in N(u)$ and some $v \in N(w) \backslash\{u\}$, swapping $\ell(u w)$ with $\ell(w v)$ results in $c_{\ell}(v) \neq 6$, then swap $\ell(u w)$ with $\ell(w v)$;

(b) else, for all $q \in N(x) \backslash\{u\}$, for all $r \in N(y) \backslash\{u\}$, and for all $s \in N(z) \backslash\{u\}$, remove the labels of the edges $x q, y r$, and $z s$, for a total of 6 labels removed. Note that two 1's, two 2's, and two 3's have been removed since each vertex in the closed neighbourhood of $u$ is incident to exactly one edge with label $j$ for all $1 \leq j \leq 3$. Then, assuming this is the $i^{t h}$ iteration of Step 4, for all $q \in N(x) \backslash\{u\}$, for all $r \in N(y) \backslash\{u\}$, and for all $s \in N(z) \backslash\{u\}$, set $\ell^{i+1}(x q)=2, \ell^{i+1}(y r)=3$, and $\ell^{i+1}(z s)=1$.

First, note that only edges incident to vertices at distance at most 2 from $u$ have their labels changed and so each execution of Step 4 deals with disjoint vertices and edges in relation to the other executions of Step 4. If Step 4(a) is executed at the $i^{t h}$ iteration, then $c_{\ell}^{i+1}(u) \neq 6$ and no other vertex changed from colour 6 to another colour or from another colour to 6. If Step 4(b) is executed at the $i^{\text {th }}$ iteration, then $c_{\ell}^{i+1}(u)=6$ remains unchanged, however, $c_{\ell}^{i+1}(x)=5, c_{\ell}^{i+1}(y)=8$, and $c_{\ell}^{i+1}(z)=5$. Moreover, since Step 4(a) was not executed, then for all $q \in N(x) \backslash\{u\}$, after the $i^{t h}$ iteration of Step 4 (specifically Step 4(b) was executed), we have $c_{\ell}^{i+1}(q) \neq c_{\ell}^{i+1}(x)$ and $c_{\ell}^{i+1}(q) \neq 6$. Indeed, let $\alpha, \beta=N(x) \backslash\{u\}$. For exactly one $q \in N(x) \backslash\{u\}$, say $\alpha$, the label of $x \alpha$ was changed from 3 to 2 . But since Step 4(a) was not executed, we have that $c_{\ell}^{i}(\alpha)-3+1=6$ and so, $c_{\ell}^{i}(\alpha)=8$. Therefore, $c_{\ell}^{i+1}(\alpha)=7$. Also since Step 4(a) was not executed, we have that $c_{\ell}^{i}(\beta)-2+1=6$ and so, $c_{\ell}^{i}(\beta)=7$. Since none of the labels incident to $\beta$ changed, $c_{\ell}^{i}(\beta)=c_{\ell}^{i+1}(\beta)=7$. Analogously, for all $r \in N(y) \backslash\{u\}$ and for all $s \in N(z) \backslash\{u\}$, we have $c_{\ell}^{i+1}(r) \neq c_{\ell}^{i+1}(y), c_{\ell}^{i+1}(r) \neq 6, c_{\ell}^{i+1}(s) \neq c_{\ell}^{i+1}(z)$, and $c_{\ell}^{i+1}(s) \neq 6$. Indeed, it is easy to check that for all $r \in N(y) \backslash\{u\}$ and for all $s \in N(z) \backslash\{u\}$, we have $c_{\ell}^{i+1}(r)=7$ and $c_{\ell}^{i+1}(s)=4$.

Note that Step 4 eventually ends since either $c_{\ell}(u) \neq 6$ or all of the neighbours of $u$ have a colour different from 6 after each execution of Step 4, no vertices change to colour 6 , and no new vertices come into conflict in terms of colour. Once, Step 4 can no longer be executed, the 3 -labelling $\ell$ is proper and equitable. Indeed, there are no more vertices in $A$ whose colour conflicts with a vertex in $B$.

Case $k \geq 4$ : the algorithm proceeds as follows:

4. While there exists a subgraph of $G$ isomorphic to $S_{k}$, with center $u$ such that $u \in A$ and for all $w \in N(u)$, we have that $c_{\ell}(u)=c_{\ell}(w)=p$ (see Figure 8),

(a) if for some $w \in N(u)$ and some $v \in N(w) \backslash\{u\}$, swapping $\ell(u w)$ with $\ell(w v)$ results in $c_{\ell}(v) \neq p$, then $\operatorname{swap} \ell(u w)$ with $\ell(w v)$;

(b) else, let $x, y \in N(u), q, r \in N(x) \backslash\{u\}$ and $s \in N(y) \backslash\{u\}$ such that, if this is the $i^{t h}$ iteration of Step $4, \ell^{i}(u x)=\Delta-1, \ell^{i}(u y)=\Delta, \ell^{i}(x q)=1, \ell^{i}(x r)=2, \ell^{i}(y s)=1$, and swap the labels of these edges so that $\ell^{i+1}(u x)=1, \ell^{i+1}(u y)=\Delta-1, \ell^{i+1}(x q)=\Delta$, $\ell^{i+1}(x r)=1$, and $\ell^{i+1}(y s)=2$ (see Figure 8 ). Note that such a labelling $\ell^{i}$ exists since each vertex in the closed neighbourhood of $u$ is incident to exactly one edge with label $j$ for all $1 \leq j \leq k$.

First, note that only edges incident to vertices at distance at most 2 from $u$ have their labels changed and so each execution of Step 4 deals with disjoint vertices and edges in relation to 


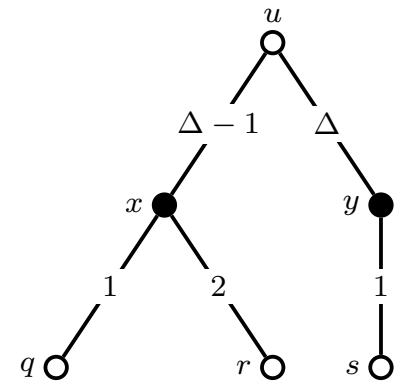

(a) Before Step 4(b) is executed.

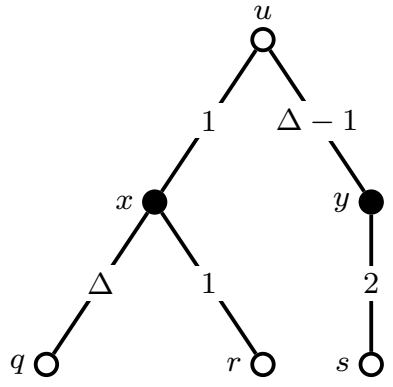

(b) After Step 4(b) is executed.

Figure 8: The case before and after Step 4(b) is executed when $k \geq 4$.

the other executions of Step 4. If Step 4(a) is executed at the $i^{\text {th }}$ iteration of Step 4, then $c_{\ell}^{i+1}(u) \neq p$ and no other vertex changed from colour $p$ to another colour or from another colour to $p$. If Step 4(b) is executed at the $i^{t h}$ iteration of Step 4, then $c_{\ell}^{i+1}(u)=p-\Delta+1 \neq p$, $c_{\ell}^{i+1}(x)=p-\Delta+1+\Delta-2+1=p, c_{\ell}^{i+1}(y)=p-\Delta+\Delta-1-1+2=p$, and since Step 4(a) was not executed, $\Delta \neq \Delta-1$, and $\Delta \neq 2$, we have that $c_{\ell}^{i+1}(q) \neq p, c_{\ell}^{i+1}(r) \neq p$, and $c_{\ell}^{i+1}(s) \neq p$.

Note that Step 4 eventually ends since $c_{\ell}(u) \neq p$ after each execution of Step 4 and no new vertices come into conflict in terms of colour. Once, Step 4 can no longer be executed, the $k$ labelling $\ell$ is proper and equitable. Indeed, there are no more vertices in $A$ whose colour conflicts with a vertex in $B$.

\section{Conclusion and open questions}

In this work, we have provided several results on equitable proper labellings, a notion that was previously introduced and studied in [1] and [11]. Answering a question of Senhaji, we proved that there exist infinitely many graphs $G$ with $\chi_{\Sigma}(G)<\overline{\chi_{\Sigma}}(G)$. Actually, unless $\mathrm{P}=\mathrm{NP}$, there is no good characterisation of the graphs with $\chi_{\Sigma}(G)=\overline{\chi_{\Sigma}}(G)$. In the bipartite case, we exhibited operations for building infinitely many bipartite graphs $G$ with $\chi_{\Sigma}(G)<\overline{\chi_{\Sigma}}(G)$. We also proved that, for every bipartite graph $G$ with $\chi_{\Sigma}(G)=3$, we have $\overline{\chi_{\Sigma}}(G)=3$. Finally, we proved that $\overline{\chi_{\Sigma}}(G) \leq k$ holds for every $k$-regular bipartite graph $G$ with $k \geq 3$.

Regarding our results, some aspects remain open.

- In particular, we still wonder whether there is a good characterisation of bipartite graphs $G$ with $2=\chi_{\Sigma}(G)<\overline{\chi_{\Sigma}}(G)=3$. Recall that all such graphs we have exhibited share very particular properties, which led to raising Question 3.8, whose aspects are very intriguing. If such a good characterisation was to not exist, then that would be an interesting contrast with the non-equitable case (regarding the characterisation of odd multi-cacti from [12]).

- Regarding Conjecture 1.2, only the case $k=3$ of our Theorem 4.2 gives a satisfying answer. A next step could be to prove Conjecture 1.2 for all $k$-regular bipartite graphs with $k \geq 4$. Recall that König's Theorem (Lemma 4.1) was a nice tool for ensuring equitability in our proof of Theorem 4.2; another interesting direction could be to consider generalisations of our approach to more families of class- 1 graphs, i.e., graphs with chromatic index equal to the maximum degree.

More generally speaking, there are still fundamental aspects of Conjecture 1.2 which we barely understand. In particular, it would be nice to provide any general constant upper bound on $\overline{\chi_{\Sigma}}$. Providing such a bound even in the bipartite case would already be something. Also, it would be interesting to know whether $K_{4}$ is the only connected graph for which $\overline{\chi_{\Sigma}}$ is more than 3 . 


\section{References}

[1] O. Baudon, M. Pilśniak, J. Przybyło, M. Senhaji, É. Sopena, M. Woźniak. Equitable neighbour-sum-distinguishing edge and total colourings. Discrete Applied Mathematics, 222:40-53, 2017.

[2] J. Bensmail, B. Li, B. Li, N. Nisse. On Minimizing the Maximum Color for the 1-2-3 Conjecture. Discrete Applied Mathematics, 289:32-51, 2021.

[3] J. Bensmail, F. Fioravantes, N. Nisse. On Proper Labellings of Graphs with Minimum Label Sum. In: Gąsieniec L., Klasing R., Radzik T. (eds) Combinatorial Algorithms. IWOCA 2020. Lecture Notes in Computer Science, vol 12126. Springer, Cham.

[4] A. Dudek, D. Wajc. On the complexity of verterx-coloring edge-weightings. Discrete Mathematics Theoretical Computer Science, 13(3):45-50, 2011.

[5] M. Kalkowski, M. Karoński, F. Pfender. Vertex-coloring edge-weightings: towards the 1-2-3 Conjecture. Journal of Combinatorial Theory, Series B, 100:347-349, 2010.

[6] M. Karoński, T. Łuczak, A. Thomason. Edge weights and vertex colours. Journal of Combinatorial Theory, Series B, 91:151-157, 2004.

[7] D. König. Über Graphen und ihre Anwendung auf Determinantentheorie und Mengenlehre. Mathematische Annalen, 77(4):453-465, 1916.

[8] W. Mulzer, G. Rote. Minimum-weight triangulation is NP-hard. Journal of the ACM, 55(2):11, 2008.

[9] J. Przybyło, M. Woźniak. On a 1,2 Conjecture. Discrete Mathematics and Theoretical Computer Science, 12(1):101-108, 2010.

[10] B. Seamone. The 1-2-3 Conjecture and related problems: a survey. Preprint, 2012. Available at http://arxiv.org/abs/1211.5122.

[11] M. Senhaji. Neighbour-distinguishing decompositions of graphs. Ph.D. thesis, University of Bordeaux, 2018. Available at https://tel.archives-ouvertes.fr/tel-01962280.

[12] C. Thomassen, Y. Wu, C.-Q. Zhang. The 3-flow conjecture, factors modulo $k$, and the 1-2-3conjecture. Journal of Combinatorial Theory, Series B, 121:308-325, 2016. 